\title{
THE ALMA SPECTROSCOPIC SURVEY IN THE HUBBLE ULTRA DEEP FIELD: MOLECULAR GAS RESERVOIRS IN HIGH-REDSHIFT GALAXIES
}

\author{
Roberto Decarli $^{1}$, Fabian Walter ${ }^{1,2,3}$, Manuel Aravena ${ }^{4}$, Chris Carilli $^{3,5}$, Rychard Bouwens ${ }^{6}$, Elisabete Da Cunha ${ }^{7,8}$, \\ Emanuele Daddi ${ }^{9}$, David Elbaz ${ }^{9}$, Dominik Riechers ${ }^{10}$, IAn SMail ${ }^{11}$, Mark Swinbank ${ }^{11}$, Axel Weiss ${ }^{12}$, Roland Bacon ${ }^{13}$, \\ Franz Bauer $^{14,15,16}$, Eric F. Bell ${ }^{17}$, Frank Bertoldi ${ }^{18}$, Scott Chapman ${ }^{19}$, Luis Colina ${ }^{20}$, Paulo C. Cortes ${ }^{21,22}$, \\ Pierre $\mathrm{Cox}^{21}$, Jorge Gónzalez-López ${ }^{23}$, Hanae Inami ${ }^{13}$, Rob Ivison ${ }^{24,25}$, Jacqueline Hodge ${ }^{6}$, Alex Karim ${ }^{18}$, \\ Benjamin Magnelli ${ }^{18}$, Kazuaki Ota ${ }^{5,26}$, Gergö Popping ${ }^{24}$, Hans-Walter Rix ${ }^{1}$, Mark Sargent ${ }^{27}$, \\ ARJEN VAN DER Wel ${ }^{1}$, AND PAUl VAN DER WERF ${ }^{6}$ \\ ${ }^{1}$ Max-Planck Institut für Astronomie, Königstuhl 17, D-69117, Heidelberg, Germany; decarli@mpia.de \\ ${ }^{2}$ Astronomy Department, California Institute of Technology, MC105-24, Pasadena, CA 91125, USA \\ ${ }^{3}$ National Radio Astronomy Observatory, Pete V. Domenici Array Science Center, P.O. Box O, Socorro, NM 87801, USA \\ ${ }^{4}$ Núcleo de Astronomía, Facultad de Ingeniería, Universidad Diego Portales, Av. Ejército 441, Santiago, Chile \\ ${ }^{5}$ Cavendish Laboratory, University of Cambridge, 19 J J Thomson Avenue, Cambridge CB3 OHE, UK \\ ${ }^{6}$ Leiden Observatory, Leiden University, P.O. Box 9513, NL2300 RA Leiden, The Netherlands \\ ${ }^{7}$ Centre for Astrophysics and Supercomputing, Swinburne University of Technology, Hawthorn, Victoria 3122, Australia \\ ${ }_{8}^{8}$ Research School of Astronomy and Astrophysics, Australian National University, Canberra, ACT 2611, Australia \\ ${ }^{9}$ Laboratoire AIM, CEA/DSM-CNRS-Universite Paris Diderot, Irfu/Service d'Astrophysique, CEA Saclay, \\ Orme des Merisiers, F-91191 Gif-sur-Yvette cedex, France \\ ${ }^{10}$ Cornell University, 220 Space Sciences Building, Ithaca, NY 14853, USA \\ ${ }^{11} 6$ Centre for Extragalactic Astronomy, Department of Physics, Durham University, South Road, Durham, DH1 3LE, UK \\ ${ }_{12}$ Max-Planck-Institut für Radioastronomie, Auf dem Hügel 69, D-53121 Bonn, Germany \\ ${ }^{13}$ Université Lyon 1, 9 Avenue Charles André, F-69561 Saint Genis Laval, France \\ ${ }^{14}$ Instituto de Astrofísica, Facultad de Física, Pontificia Universidad Católica de Chile Av. Vicuña Mackenna 4860, 782-0436 Macul, Santiago, Chile \\ ${ }^{15}$ Millennium Institute of Astrophysics (MAS), Nuncio Monseñor Sótero Sanz 100, Providencia, Santiago, Chile \\ ${ }^{16}$ Space Science Institute, 4750 Walnut Street, Suite 205, Boulder, CO 80301, USA \\ ${ }^{17}$ Department of Astronomy, University of Michigan, 1085 South University Avenue, Ann Arbor, MI 48109, USA \\ ${ }^{8}$ Argelander Institute for Astronomy, University of Bonn, Auf dem Hügel 71, D-53121 Bonn, Germany \\ 19 Dalhousie University, Halifax, Nova Scotia, Canada \\ ${ }^{20}$ ASTRO-UAM, UAM, Unidad Asociada CSIC, Spain \\ ${ }^{21}$ Joint ALMA Observatory-ESO, Av. Alonso de Córdova, 3104, Santiago, Chile \\ ${ }^{22}$ National Radio Astronomy Observatory, 520 Edgemont Road, Charlottesville, VA 22903, USA \\ ${ }^{23}$ Instituto de Astrofísica, Facultad de Física, Pontificia Universidad Católica de Chile Av. Vicuña Mackenna 4860, $782-0436$ Macul, Santiago, Chile \\ ${ }^{24}$ European Southern Observatory, Karl-Schwarzschild-Strasse 2, D-85748, Garching, Germany \\ ${ }^{25}$ Institute for Astronomy, University of Edinburgh, Royal Observatory, Blackford Hill, Edinburgh EH9 3HJ, UK \\ ${ }^{26}$ Kavli Institute for Cosmology, University of Cambridge, Madingley Road, Cambridge CB3 OHA, UK \\ ${ }^{27}$ Astronomy Centre, Department of Physics and Astronomy, University of Sussex, Brighton, BN1 9QH, UK \\ Received 2016 May 3; revised 2016 September 5; accepted 2016 September 6; published 2016 December 8
}

\begin{abstract}
We study the molecular gas properties of high- $z$ galaxies observed in the ALMA Spectroscopic Survey (ASPECS) that targets an $\sim 1 \mathrm{arcmin}^{2}$ region in the Hubble Ultra Deep Field (UDF), a blind survey of CO emission (tracing molecular gas) in the 3 and $1 \mathrm{~mm}$ bands. Of a total of 1302 galaxies in the field, 56 have spectroscopic redshifts and correspondingly well-defined physical properties. Among these, 11 have infrared luminosities $L_{\mathrm{IR}}>10^{11} L_{\odot}$, i.e., a detection in $\mathrm{CO}$ emission was expected. Out of these, 7 are detected at various significance in $\mathrm{CO}$, and 4 are undetected in $\mathrm{CO}$ emission. In the $\mathrm{CO}$-detected sources, we find $\mathrm{CO}$ excitation conditions that are lower than those typically found in starburst/sub-mm galaxy/QSO environments. We use the CO luminosities (including limits for non-detections) to derive molecular gas masses. We discuss our findings in the context of previous molecular gas observations at high redshift (star formation law, gas depletion times, gas fractions): the CO-detected galaxies in the UDF tend to reside on the low- $L_{\mathrm{IR}}$ envelope of the scatter in the $L_{\mathrm{IR}}-L_{\mathrm{CO}}^{\prime}$ relation, but exceptions exist. For the CO-detected sources, we find an average depletion time of $\sim 1 \mathrm{Gyr}$, with significant scatter. The average molecularto-stellar mass ratio $\left(M_{\mathrm{H} 2} / M_{*}\right)$ is consistent with earlier measurements of main-sequence galaxies at these redshifts, and again shows large variations among sources. In some cases, we also measure dust continuum emission. On average, the dust-based estimates of the molecular gas are a factor $\sim 2-5 \times$ smaller than those based on CO. When we account for detections as well as non-detections, we find large diversity in the molecular gas properties of the high-redshift galaxies covered by ASPECS.
\end{abstract}

Key words: galaxies: evolution - galaxies: ISM - galaxies: star formation - galaxies: statistics - instrumentation: interferometers - submillimeter: galaxies

\section{INTRODUCTION}

Molecular gas observations of galaxies throughout cosmic time are fundamental for understanding the cosmic history of the formation and evolution of galaxies (see reviews by
Kennicutt \& Evans 2012; Carilli \& Walter 2013). The molecular gas provides the fuel for star formation, thus by characterizing its properties we place quantitative constraints 
on the physical processes that lead to the stellar mass growth of galaxies. This has been a demanding task in terms of telescope time. To date, only some several hundred sources at $z>1$ have been detected in a molecular gas tracer (typically the rotational transitions of the carbon monoxide ${ }^{12} \mathrm{CO}$ molecule; e.g., Carilli \& Walter 2013). This sample is dominated by "extreme" sources, such as QSO host galaxies (e.g., Bertoldi et al. 2003; Walter et al. 2003; Weiß et al. 2007; Wang et al. 2013) or submillimeter galaxies (e.g., Frayer et al. 1998; Neri et al. 2003; Greve et al. 2005; Bothwell et al. 2013; Riechers et al. 2013; Aravena et al. 2016), with IR luminosities $L_{\mathrm{IR}} \gg 10^{12} L_{\odot}$ and star formation rates (SFRs) $\gg 100$ $M_{\odot} \mathrm{yr}^{-1}$. These extreme sources might contribute significantly to the star formation budget in the universe at $z>4$, but their role declines with cosmic time (Casey et al. 2014). Indeed, the bulk of star formation up to $z \sim 2$ is observed in galaxies along the so-called "main sequence" (Elbaz et al. 2007, 2011; Noeske et al. 2007; Daddi et al. 2010a, 2010b; Genzel et al. 2010; Wuyts et al. 2011; Whitaker et al. 2012), a tight (scatter rms $\sim 0.3$ dex) relation between the SFR and the stellar mass, $M_{*}$. Addressing the molecular gas content of main-sequence galaxies beyond the local universe has become feasible only in recent years.

The first step in the characterization of the molecular gas content of galaxies is the measure of the molecular gas mass, $M_{\mathrm{H} 2}$. The ${ }^{12} \mathrm{CO}$ molecule (hereafter, $\mathrm{CO}$ ) is the second most abundant molecule in the universe, and it is relatively easy to target thanks to its bright rotational transitions. The use of $\mathrm{CO}$ as a tracer for the molecular gas mass requires assuming a conversion factor, $\alpha_{\mathrm{CO}}$, to pass from $\mathrm{CO}(1-0)$ luminosities to $\mathrm{H}_{2}$ masses. At $z \sim 0$, the conversion factor that is typically used is $\sim 4 M_{\odot}\left(\mathrm{K} \mathrm{km} \mathrm{s}^{-1} \mathrm{pc}^{2}\right)^{-1}$ for "normal" $M_{*}>10^{9} M_{\odot}$ starforming galaxies with metallicities close to solar (see Bolatto et al. 2013, for a recent review). If other $\mathrm{CO}$ transitions are observed instead of the $J=1 \rightarrow 0$ ground state transition, a further factor is required to account for the $\mathrm{CO}$ excitation (see, e.g., Weiß et al. 2007; Daddi et al. 2015). Tacconi et al. (2010) and Daddi et al. (2010a) investigated the molecular gas content of highly star-forming galaxies at $z \sim 1.2$ and $z \sim 2.3$ via the $\mathrm{CO}(3-2)$ transition. They found large reservoirs of gas, yielding molecular-to-stellar mass ratios $M_{\mathrm{H} 2} / M_{*} \sim 1$. These values are significantly higher than those observed in local galaxies $(\sim 0.1$, see, e.g., Leroy et al. 2008), suggesting a strong evolution of $M_{\mathrm{H} 2} / M_{*}$ with redshift (see also Riechers et al. 2010; Casey et al. 2011; Geach et al. 2011; Aravena et al. 2012, 2016; Magnelli et al. 2012; Bothwell et al. 2013; Saintonge et al. 2013; Tacconi et al. 2013; Chapman et al. 2015a; Genzel et al. 2015).

An alternative approach to estimate gas masses is via dust emission. The dust mass in a galaxy can be retrieved via the study of its rest-frame submillimeter spectral energy distribution (SED) (e.g., Magdis et al. 2011, 2012; Magnelli et al. 2012; Santini et al. 2014; Béthermin et al. 2015; Berta et al. 2016), in particular via the Rayleigh-Jeans tail, which is less sensitive to the dust temperature (see, e.g., Scoville et al. 2014; Groves et al. 2015). Using the dust as a proxy of the molecular gas does not require assumptions on $\mathrm{CO}$ excitation and on $\alpha_{\mathrm{CO}}$. However, this approach relies on the assumption of a dust-to-gas mass ratio (DGR), which typically depends on the gas metallicity (Bolatto et al. 2013; Sandstrom et al. 2013; Genzel et al. 2015). Recent ALMA results report substantially lower values of $M_{\text {gas }}$ than what are typically obtained in CObased studies (Scoville et al. 2014, 2016).
In the present paper, we study the molecular gas properties of galaxies in ASPECS, the ALMA Spectroscopic Survey in the Hubble Ultra Deep Field (UDF). This is a blind search for CO emission using the Atacama Large Millimeter/submillimeter Array (ALMA). The goal is to constrain the molecular gas content of an unbiased sample of galaxies. The targeted region is one of the best-studied areas of the sky, with exquisitely deep photometry in $>25$ X-ray-to-far-infrared (IR) bands, photometric redshifts, and dozens of spectroscopic redshifts. This provides us with an exquisite wealth of ancillary data, which is instrumental to place our $\mathrm{CO}$ measurements in the context of galaxy properties. Thanks to the deep-field nature of our approach, we avoid potential biases related to the preselection of targets, and include both detections and nondetections in our analysis. Our data set combines $3 \mathrm{~mm}$ and $1 \mathrm{~mm}$ observations of the same galaxies, thus providing constraints on the $\mathrm{CO}$ excitation. Furthermore, the combination of the spectral line survey and the $1 \mathrm{~mm}$ continuum image allows us to compare CO- and dust-based estimates of the gas mass. In other papers of this series, we present the data set and the catalog of blindly selected CO emitters (Paper I, Walter et al. 2016), we study the properties of $1.2 \mathrm{~mm}$ detected sources (Paper II, Aravena et al. 2016a), we discuss the inferred constraints on the luminosity functions of CO (Paper III, Decarli et al. 2016a), and we search for [C II] emission in $z=6-8$ galaxies (Paper V, Aravena et al. 2016b). Paper VI (Bouwens et al. 2016) places our findings in the context of the dust extinction law for $z>2$ galaxies, and Paper VII (Carilli et al. 2016) uses ASPECS to place first direct constraints on intensity mapping experiments. Here we place the $\mathrm{CO}$ detections in the context of the properties of the associated galaxies. In Section 2 we summarize the observational data set; in Section 3 we describe our sample; in Section 4 we present CO-based measurements, which are discussed in the context of galaxy properties in Section 5. We summarize our findings in Section 6.

Throughout the paper we assume a standard $\Lambda \mathrm{CDM}$ cosmology with $H_{0}=70 \mathrm{~km} \mathrm{~s}^{-1} \mathrm{Mpc}^{-1}, \Omega_{\mathrm{m}}=0.3$, and $\Omega_{\Lambda}=0.7$ (broadly consistent with the measurements in Planck Collaboration XIII 2016), and a Chabrier (2003) initial stellar mass function. Magnitudes are expressed in the AB photometric system (Oke \& Gunn 1983).

\section{OBSERVATIONS}

\subsection{The ALMA Data Set}

The details of the ALMA data set (observations and data reduction) are presented in Paper I of this series. Here we briefly summarize the observational details that are relevant for the present study. The data set consists of two frequency scans in ALMA band $3(3 \mathrm{~mm}, 84-115 \mathrm{GHz})$ and in band $6(1 \mathrm{~mm}$, $212-272 \mathrm{GHz})$. In the case of the $3 \mathrm{~mm}$ observations, we obtained a single pointing centered at R.A. $=03: 32: 37.900$, decl. $=-27: 46: 25.00(\mathrm{~J} 2000.0)$, close to the northern corner of the Hubble eXtremely Deep Field (XDF, Illingworth et al. 2013). The primary beam has a diameter of $\approx 65^{\prime \prime}$ at the central frequency of the band $(99.5 \mathrm{GHz})$. The typical noise $\mathrm{rms}$ is $\sim 0.18 \mathrm{mJy} \mathrm{beam}^{-1}$ per $50 \mathrm{~km} \mathrm{~s}^{-1}$ channel. The $1 \mathrm{~mm}$ observations consist of a seven-pointing mosaic covering approximately the same area as the $3 \mathrm{~mm}$ observations. The typical noise rms is $0.44 \mathrm{mJy}_{\text {beam }}^{-1}$ per $50 \mathrm{~km} \mathrm{~s}^{-1}$ channel. 
The resulting $1 \mathrm{~mm}$ continuum image reaches a noise rms of $12.7 \mu \mathrm{Jy}$ beam $^{-1}$ at the center of the mosaic (see Paper II).

\subsection{Ancillary Data}

We complement the ALMA data with X-ray-to-far-IR photometry from public catalogs of this field, as well as optical/near-IR spectroscopic information where available. The main sources for the photometry are the compilations by Coe et al. (2006) and Skelton et al. (2014). The former includes optical photometry based on the original Hubble Space Telescope (HST) Advanced Camera for Survey (ACS) images of the Hubble UDF (Beckwith et al. 2006) and near-IR images obtained with HST NICMOS. The latter also compiles optical/ near-IR observations with HST ACS and the Wide Field Camera 3 (WFC3) from the Hubble XDF (Illingworth et al. 2013), Spitzer IRAC, as well as a wealth of ground-based optical/nearIR observations. Spitzer MIPS data at 24 and $70 \mu \mathrm{m}$, as well as Herschel PACS and SPIRE data come from the work by Elbaz et al. (2011). X-ray data are taken from the Extended Chandra Deep Field South Survey (Lehmer et al. 2005) and from the Chandra Source Catalog (Evans et al. 2010).

Photometric redshifts $\left(z_{\text {photo }}\right)$ are available for all of the optically selected sources in the field. At a limiting magnitude $i=28 \mathrm{mag}$, the median uncertainty is $\delta z_{\text {photo }} \sim 0.5$, and it reaches $\delta z_{\text {photo }} \sim 1$ at $i \approx 30 \mathrm{mag}$ (Coe et al. 2006). The compilation of Skelton et al. (2014) provides even more robust photometric redshifts, thanks to the expanded photometric data set. The agreement with available spectroscopic redshifts is typically very good in these cases, with a standard deviation on $\Delta z /(1+z)$ of $\approx 0.01$ (Skelton et al. 2014). In addition, the 3D$H S T$ survey provides HST ACS and WFC3 grism observations of the field, yielding grism redshifts for tens of sources in our pointing (Momcheva et al. 2016). Slit spectroscopy for 74 (mostly bright) galaxies in the field is also available (Le Fèvre et al. 2005; Skelton et al. 2014; Morris et al. 2015). Finally, integral field spectroscopy of this field has been secured with the ESO Very Large Telescope/MUSE. These data are part of a Guaranteed Time observing program targeting the UDF. In particular, a single ( $\left.1 \mathrm{arcmin}^{2}\right)$ deep ( $21 \mathrm{hr}$ on source) pointing overlaps with $\sim 70 \%$ of the ASPECS coverage. The cubes have been processed and analyzed with the improved MUSE GTO pipeline. These observations will be presented in R. Bacon et al. (2016, in preparation).

Within a radius of $34^{\prime \prime}$ from the ALMA $3 \mathrm{~mm}$ pointing center (approximately the size of the primary beam of our $3 \mathrm{~mm}$ observations), there are 1302 galaxies from the combination of all the available photometric catalogs. We use the high- $z$ extension of MAGPHYS (da Cunha et al. 2008, 2015) to fit the SEDs of all of them. The input photometry includes 26 broad and medium filters ranging from observed $U$ band to Spitzer IRAC $8 \mu \mathrm{m}$. Additionally, we include the ASPECS $1 \mathrm{~mm}$ continuum photometry for those sources where $>2-\sigma$ emission is reported in our $1 \mathrm{~mm}$ continuum image. We do not include any Spitzer MIPS or Herschel PACS photometry because the angular resolutions of these instruments are not sufficient to accurately pinpoint the emission. ${ }^{28}$ Our MAGPHYS analysis

\footnotetext{
${ }^{28}$ For instance, including MIPS and PACS in the SED fits yields an overestimate (by a factor $\sim 3$ ) of the SFR in the brightest source in our sample, but with a poor SED fit quality, because of the contamination of foreground sources; on the other hand, the second brightest galaxy, which appears isolated, shows consistent results regardless of whether the fit is performed with or without MIPS and PACS photometry.
}

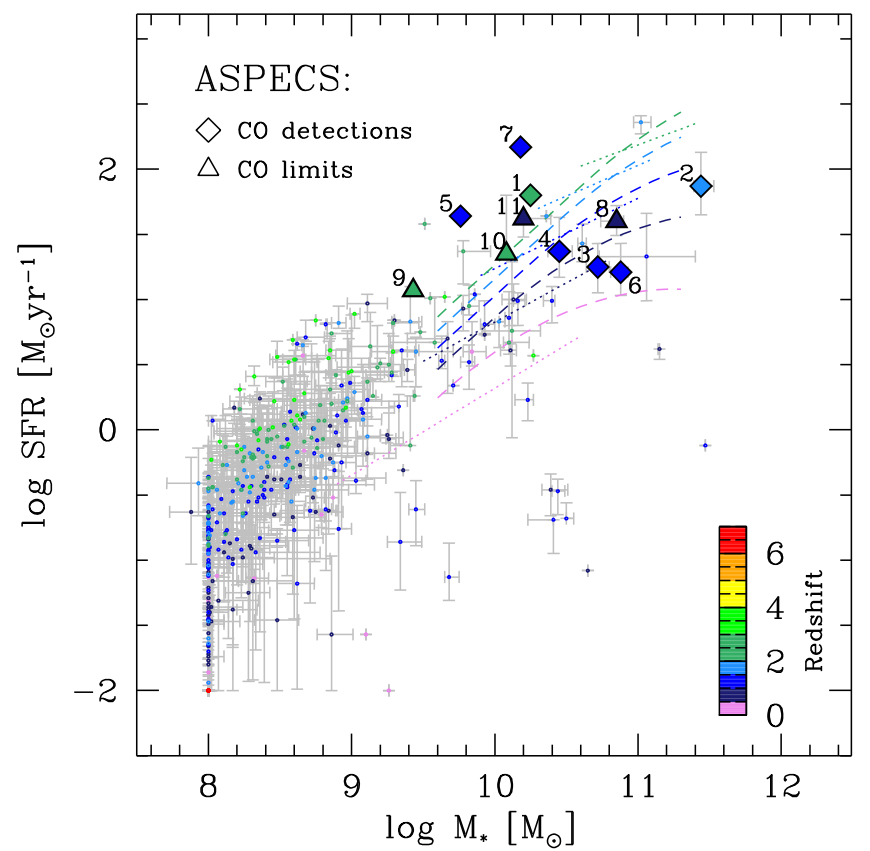

Figure 1. Star formation rates and stellar masses of all the galaxies in our field, color-coded by redshift. Inferred parameters are derived using the high- $z$ extension of MAGPHYS (da Cunha et al. 2008, 2015). The sample discussed here is highlighted with large symbols: diamonds refer to the CO detections, while galaxies in the present sample that are not detected in $\mathrm{CO}$ are marked with triangles. We stress that only galaxies with secure spectroscopic redshifts are considered in the present analysis. The loci of the main sequence in various redshift bins are shown as dotted lines (from Whitaker et al. 2012) and dashed lines (from Schreiber et al. 2015). Half of the galaxies in our sample lie along the main sequence at their respective redshifts. ID.5, 7, and 11 occupy the "starburst" region above the main sequence, while ID.3 and 6 exhibit an SFR $\sim 3 \times$ lower than what is typically observed in main-sequence galaxies at the same redshifts and stellar masses.

provides us with a posterior probability distribution of the stellar mass $\left(M_{*}\right)$, the SFR, the specific SFR ( $\mathrm{SSFR}=\mathrm{SFR} /$ $\left.M_{*}\right)$, the dust mass $\left(M_{\text {dust }}\right)$, and the IR luminosity for each galaxy in the field. We take the $14 \%$ and $86 \%$ quartiles of the posterior distributions as the uncertainties in the parameters, and we account for an additional fiducial $10 \%$ uncertainty that is due to systematics (subtleties in the photometric analysis adopted in the input catalogs, such as aperture corrections and deblending assumptions; zero-point uncertainties; etc.). Figure 1 shows the SFR as a function of $M_{*}$ for all the 1302 galaxies in our field.

\section{THE SAMPLE}

We focus our discussion on those galaxies in the field covered by ASPECS that we originally expected to detect in $\mathrm{CO}$ emission. Our expectations are based on the MAGPHYS predictions discussed in the previous section. Figure 1 shows the stellar masses and SFRs of all 1302 galaxies in the field (color-coded by redshift). Out of these, 56 galaxies have secure spectroscopic redshifts within our CO redshift coverage, and are brighter than $27.5 \mathrm{mag}$ in the filters F850LW or F105W ( $z$ and $Y$ bands, respectively). ${ }^{29} \mathrm{We}$ further restrict our analysis to the redshift windows for which ASPECS covers at least one of

\footnotetext{
${ }^{29}$ This flux cut allows us to reject sources that are too faint for a reliable SED analysis.
} 
Table 1

Sample of Galaxies Examined in this Work, and Their Optical/near-IR Global Properties

\begin{tabular}{|c|c|c|c|c|c|c|c|c|c|c|}
\hline (1) & $\begin{array}{c}\text { ASPECS } \\
\text { Name } \\
(2)\end{array}$ & $\begin{array}{c}\text { Optical R.A. } \\
\text { (3) }\end{array}$ & $\begin{array}{c}\text { Optical decl. } \\
\text { (4) }\end{array}$ & (5) & $\begin{array}{c}\text { Obs.CO } \\
\text { Trans. } \\
(6)\end{array}$ & $\begin{array}{r}M_{*} \\
\left(\times 10^{9} \quad M_{\odot}\right) \\
(7)\end{array}$ & $\begin{array}{r}\mathrm{SFR} \\
\left(M_{\odot} \mathrm{yr}^{-1}\right) \\
(8)\end{array}$ & $\begin{array}{r}\text { sSFR } \\
\left(\mathrm{Gyr}^{-1}\right) \\
(9)\end{array}$ & $\left.\begin{array}{r}L_{\mathrm{IR}} \\
\left(\times 10^{11} L_{\odot}\right. \\
\quad(10)\end{array}\right)$ & $\begin{array}{c}R_{\mathrm{e}} \\
(\mathrm{kpc}) \\
(11)\end{array}$ \\
\hline 1 & $3 \mathrm{~mm} \cdot 1^{\mathrm{a}}, \mathrm{C} 1$ & $03: 32: 38.54$ & $-27: 46: 34.0$ & 2.543 & $3,7,8$ & $17.8_{-1.7}^{+1.8}$ & $63_{-6}^{+6}$ & $3.4_{-0.31}^{+0.34}$ & $12.3_{-1.1}^{+1.2}$ & 1.7 \\
\hline 3 & $3 \mathrm{~mm} \cdot 3$ & 03:32:35.55 & $-27: 46: 25.5$ & 1.382 & 2,5 & $52_{-10}^{+12}$ & $18_{-7}^{+9}$ & $0.42_{-0.25}^{+0.13}$ & $1.9_{-0.9}^{+1.3}$ & 8.3 \\
\hline 4 & $3 \mathrm{~mm} .5$, C6 & $03: 32: 35.48$ & $-27: 46: 26.5$ & 1.088 & 2,4 & $28_{-5}^{+7}$ & $23_{-9}^{+20}$ & $0.9_{-0.4}^{+0.9}$ & $2.8_{-1.2}^{+2.4}$ & 5.8 \\
\hline 5 & $\ldots$ & $03: 32: 36.43$ & $-27: 46: 31.8$ & 1.098 & 2,4 & $5.8_{-0.5}^{+0.6}$ & $44_{-4}^{+4}$ & $7.41_{-0.7}^{+0.7}$ & $15.5_{-1.4}^{+1.5}$ & 6.0 \\
\hline 7 & $\ldots$ & 03:32:39.08 & $-27: 46: 01.8$ & 1.221 & 2,5 & $15.1_{-1.4}^{+1.5}$ & $148_{-13}^{+15}$ & $9.3_{-0.8}^{+0.9}$ & $49.0_{-4.4}^{+4.9}$ & 0.7 \\
\hline 8 & & 03:32:36.66 & $-27: 46: 31.0$ & 0.999 & 4 & $70_{-17}^{+11}$ & $40_{-9}^{+14}$ & $0.54_{-0.05}^{+0.40}$ & $7.1_{-2.5}^{+1.5}$ & 6.6 \\
\hline 9 & $\ldots$ & 03:32:39.41 & $-27: 46: 22.4$ & 2.447 & $3,7,8$ & $2.6_{-0.2}^{+0.3}$ & $11.8_{-1.1}^{+1.2}$ & $4.2_{-0.4}^{+0.4}$ & $1.35_{-0.12}^{+0.13}$ & 5.8 \\
\hline 10 & $\ldots$ & 03:32:37.07 & $-27: 46: 17.2$ & 2.224 & $3,6,7$ & $12.0_{-1.2}^{+1.2}$ & $22_{-2}^{+41}$ & $1.86_{-0.17}^{+3.53}$ & $1.95_{-0.18}^{+5.3}$ & 2.7 \\
\hline 11 & $\ldots$ & $03: 32: 36.33$ & $-27: 46: 00.1$ & 0.895 & 4 & $15.9_{-1.4}^{+9.0}$ & $42_{-12}^{+4}$ & $2.7_{-1.5}^{+0.27}$ & $5.8_{-1.6}^{+0.6}$ & 1.2 \\
\hline
\end{tabular}

Notes. Sorting is based on the significance of the CO detection. (1) Source ID. (2) ASPECS name for blind CO detections (3 mm.X, see Paper I) and for the blind $1.2 \mathrm{~mm}$ continuum detections (CX, see Paper II). (3)-(4) Optical coordinates in Skelton et al. (2014). (5) Redshift. (6) $J_{\text {up }}$ of the CO transitions encompassed in our ASPECS Data. (7)-(10) MAGPHYS-derived stellar mass $\left(M_{*}\right)$, star formation rate (SFR), specific star formation rate (sSFR), IR luminosity ( $\left.L_{\mathrm{IR}}\right)$. (11) Effective radius from the near-IR analysis by van der Wel et al. (2012).

${ }^{\text {a Also }} 1 \mathrm{~mm} .1$ and $1 \mathrm{~mm} .2$, see Paper I.
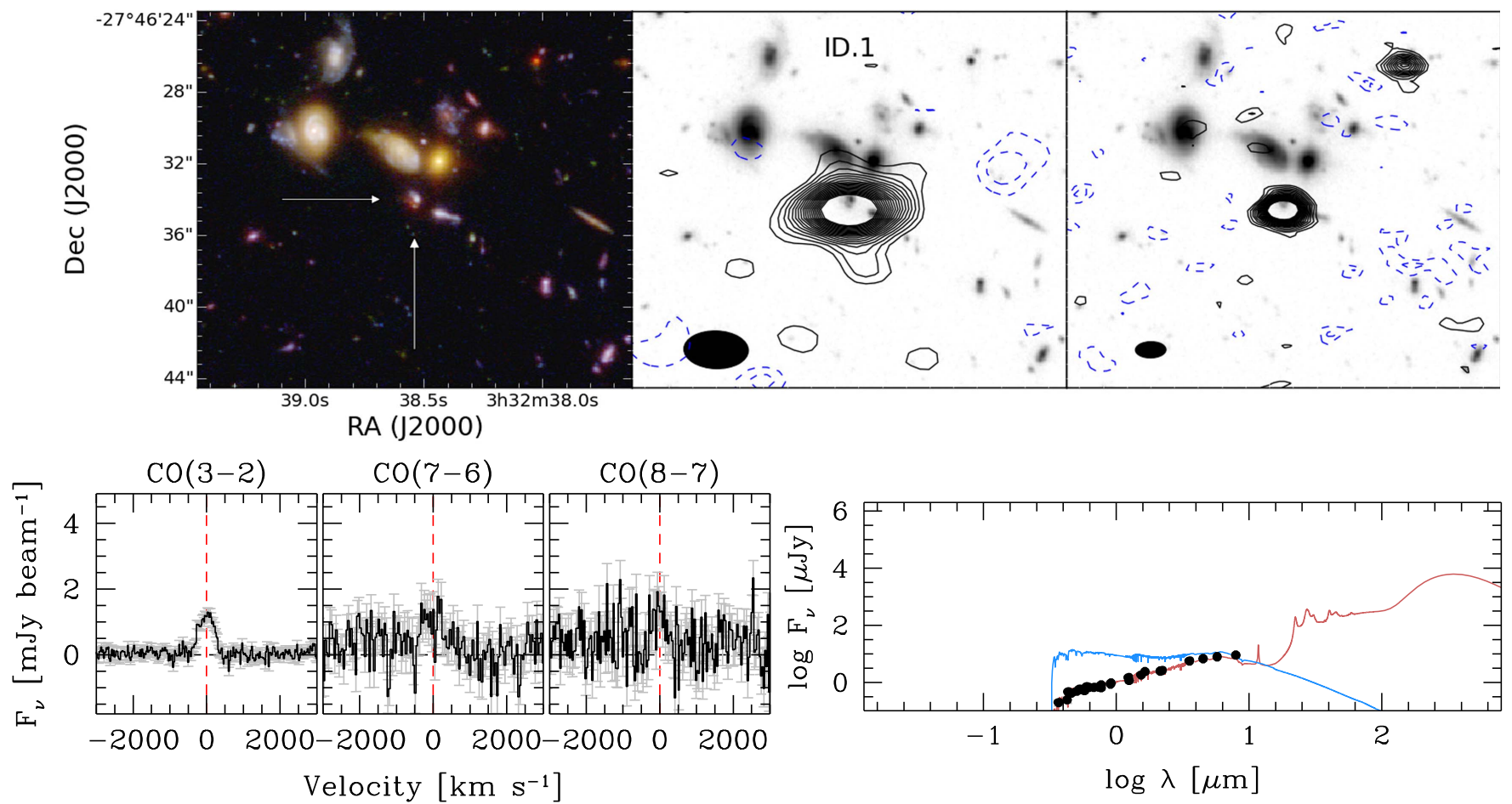

Figure 2. Top left: HST F105W/F775W/F435W RGB image of ID.1. The postage stamp is $20^{\prime \prime} \times 20^{\prime \prime}$. Top center: HST F125W image of the same field. The map of the lowest- $J$ accessible $\mathrm{CO}$ transition (in this case, $\mathrm{CO}[3-2]$ ) is shown as contours $\left( \pm 2,3, \ldots, 20-\sigma\left[\sigma(\mathrm{ID} .1)=0.78 \mathrm{mJy}^{-1}\right.\right.$ beam ${ }^{-1}$; solid black lines for the positive isophotes, dashed blue lines for the negative). The synthesized beam is shown as a black ellipse. Top right: same as in the center, showing the $1.2 \mathrm{~mm}$ dust continuum. Bottom left: spectra of the CO lines encompassed in our spectral scan. Bottom right: spectral energy distribution. The red line shows the best MAGPHYS fit of the available photometry (black points), while the blue line shows the corresponding model for the unobscured stellar component. The main output parameters are quoted. Similar plots for all the sources in our sample are available in this paper.

the following low- $J$ CO transitions: $J=2 \rightarrow 1, J=3 \rightarrow 2$, or $J=4 \rightarrow 3$.

From these galaxies, we select the 11 galaxies for which the MAGPHYS SED analysis yields an IR luminosity $L_{\mathrm{IR}}>10^{11}$ $L_{\odot}$ at $>1 \sigma$ significance. These sources are marked by symbols in Figure 1, and spectroscopic redshifts are available for all of these sources. The IR luminosity of a galaxy (derived from the SED fitting) has been found to correlate with the CO luminosity (see also Section 5.2.1). Following the best fit of the relation in Carilli \& Walter (2013), the IR-luminosity cut above corresponds to $L_{\mathrm{CO}(1-0)}^{\prime}>3 \times 10^{9} \mathrm{~K} \mathrm{~km} \mathrm{~s}^{-1} \mathrm{pc}^{2}$, i.e., it is similar to the line luminosity limit of our survey (see Paper I). Consequently, we should be able to detect $\mathrm{CO}$, or at least place meaningful limits, on these 11 galaxies. 

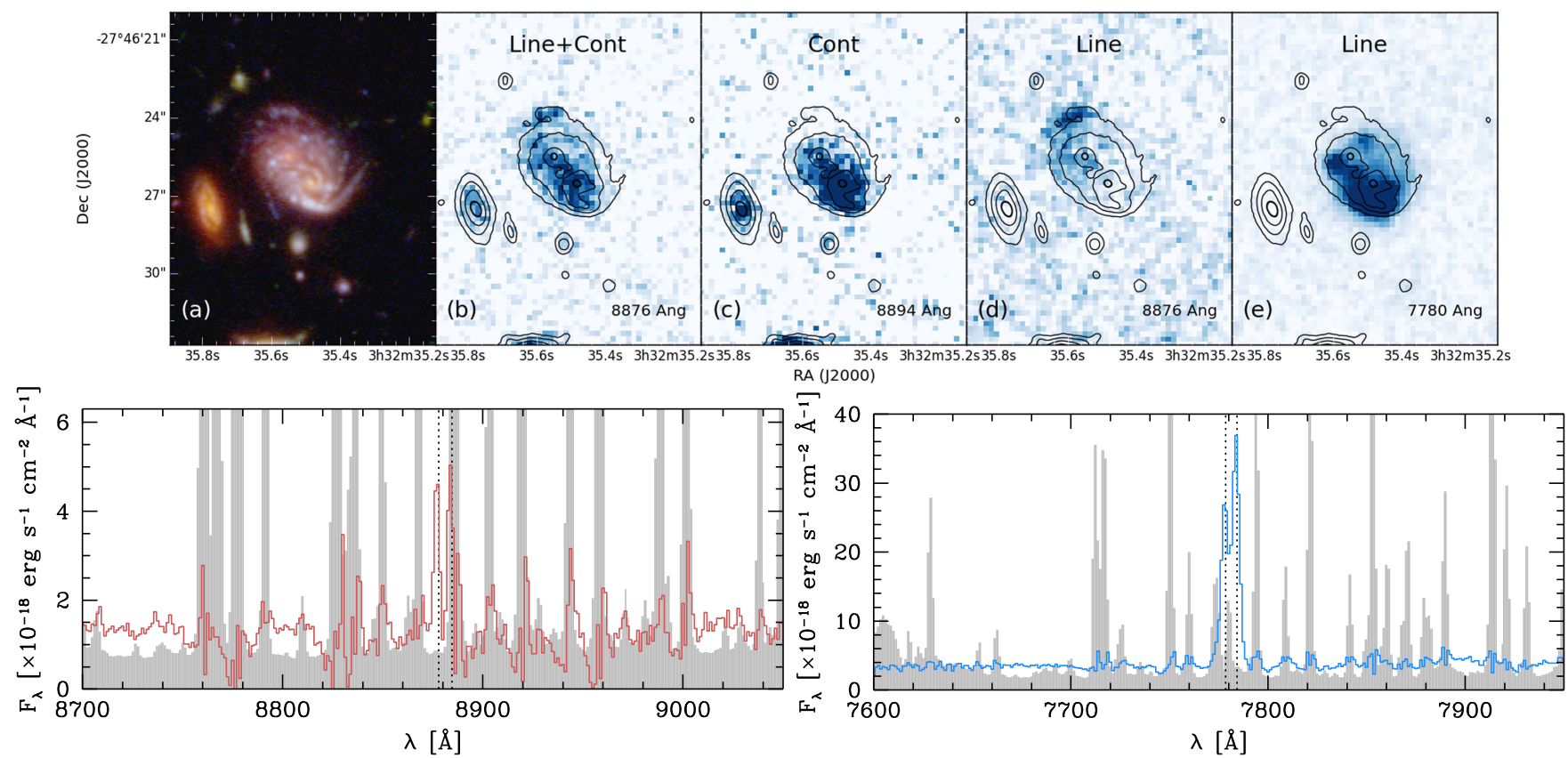

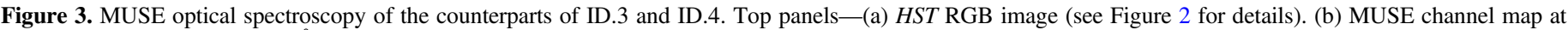

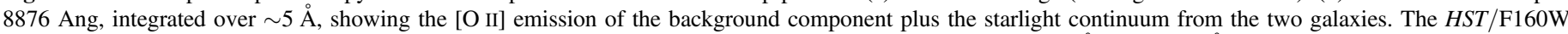

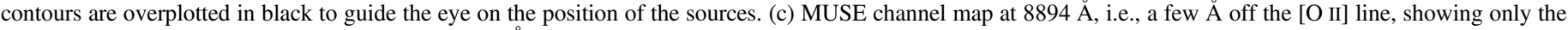

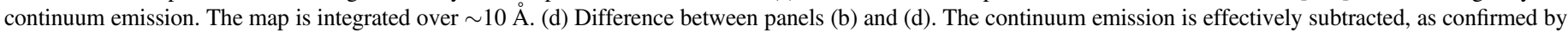

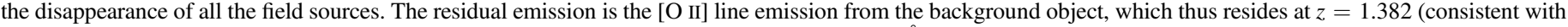

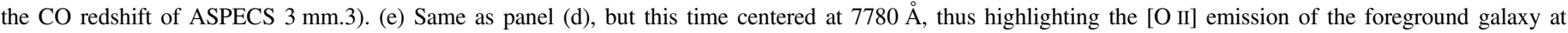

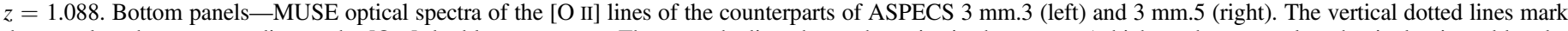

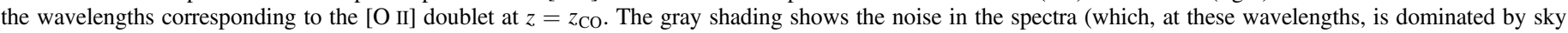
emission lines). The [O II] emission is clearly seen in both sources.

Table 1 summarizes the main optical/near-IR properties of the galaxies considered in this paper. In Figure 2 we show for one of the sources the HST image compared with the $\mathrm{CO}$ and dust continuum maps, the CO spectra, and the SED data and modeling. Similar plots are presented for all sample galaxies in this paper.

Four of the galaxies in our sample match some of the $\mathrm{CO}$ lines identified in our blind search (see Paper I). The ASPECS name for these sources is also reported in Table 1. Three additional galaxies show $\mathrm{CO}$ emission, although at lower significance. Finally, four sources remain undetected in CO.

\subsection{Notes on Individual Galaxies}

ID.1 (Table 1, Figures 2 and 11) is a compact galaxy at $z \approx 2.5$. Momcheva et al. (2016) report a grism redshift $z=2.561$, based on the detection of the [O II] line in the 3DHST data. This redshift is improved by our blind detection of three CO transitions (ASPECS $3 \mathrm{~mm} .1,1 \mathrm{~mm} .1,1 \mathrm{~mm} .2$; see Paper I), clearly pininng down the redshift to $z=2.543$. The HST images show a blue component in the north and a red component in the south (or possibly a single relatively blue component that is partially reddened in the south by a thick dust lane). A group of bright galaxies is present a few arcsec north of this galaxy, but their spectroscopic redshifts show that the group is in the foreground, with only one other source lying at $z \sim 2.5$ (the galaxy $\sim 2^{\prime \prime}$ west of ID.1). The starlight emission coincident with the $\mathrm{CO}$ detection is compact, with a scale radius $R_{e} \approx 1.7 \mathrm{kpc}$ (van der Wel et al. 2012). Chandra reveals X-ray emission associated with this galaxy. The measured X-ray flux is $F_{\mathrm{X}}=5.7 \times 10^{-17} \mathrm{erg} \mathrm{s}^{-1} \mathrm{~cm}^{-2}$, yielding an X-ray luminosity $L_{\mathrm{X}}=3.0 \times 10^{42} \mathrm{erg} \mathrm{s}^{-1}$ (Xue et al. 2011).

ID.2 (Figure 11) has an HST morphology consistent with a large disk galaxy at $z=1.552$. Its slit redshift $(z=1.552$, Kurk et al. 2013) matches our CO line detection well (ASPECS $3 \mathrm{~mm} .2$ ), assuming $\mathrm{CO}(2-1)$. The disk has an inclination of $\sim 60^{\circ}$ (based on the aspect ratio, van der Wel et al. 2012), with an effective radius of $8.3 \mathrm{kpc}$. The galaxy is detected with Chandra. Xue et al. (2011) report a flux of $F_{\mathrm{X}}=3.6 \times 10^{-15} \mathrm{erg} \mathrm{s}^{-1} \mathrm{~cm}^{-2}$ (but $2.6 \times 10^{-15} \mathrm{erg} \mathrm{s}^{-1} \mathrm{~cm}^{-2}$ in Lehmer et al. 2005), yielding an X-ray luminosity $L_{\mathrm{X}}=5.5 \times 10^{43} \mathrm{erg} \mathrm{s}^{-1}$, suggesting that ID.2 hosts an active galactic nucleus (AGN).

ID.3 and ID.4 (Figure 12) are the two components of an apparent pair of overlapping spiral galaxies. The southern component exibits bright [O II] emission at $\sim 7784 \AA$ (see Figure 3), clearly placing it at $z=1.088$ (in agreement with the CO redshift of ASPECS $3 \mathrm{~mm} .5$ ); the northern component shows bright $\mathrm{CO}$ emission (ASPECS $3 \mathrm{~mm} .3$ ), which could be interpreted as $\mathrm{CO}(2-1)$ at $z=1.382$. Our careful analysis of the MUSE data around $8880 \AA$ reveals faint [O II] emission (although contaminated by sky line emission), supporting the CO identification (see Figure 3). The disk of ID.4 has a scale radius of $5.8 \mathrm{kpc}$ based on $H S T$ imaging (van der Wel et al. 2012); for ID.3, the estimated radius is $8.3 \mathrm{kpc}$ (but the overlap with the southern component may partially affect this estimate). ID.4 appears as an upper limit in the X-ray catalog by Xue et al. (2011) $\left(F_{\mathrm{X}}<6.7 \times 10^{-17} \mathrm{erg} \mathrm{s}^{-1} \mathrm{~cm}^{-2}\right.$, $\left.L_{\mathrm{X}}<4.3 \times 10^{41} \mathrm{erg} \mathrm{s}^{-1}\right)$. 
ID.5 (Figure 13) and ID.8 (Figure 14) lie in a crowded region of our field. Skelton et al. (2014) report a spectroscopic redshift $z=1.047$ for ID.5. However, the inspection of the MUSE data reveals two clearly distinguished line sets of the [O II] doublet at $z=1.038$ and $z=1.098$. The latter matches the redshift of two CO lines that are slightly too faint to be selected in our blind search $(\mathrm{S} / \mathrm{N} \approx 4.8$, see Paper I). ID.8, on the other hand, is found at another redshift $(z=0.999)$. No CO emission is found at this position and frequency, although the lowest- $J$ transition that we encompass is $\mathrm{CO}(4-3)$ at $1 \mathrm{~mm}$. ID. 8 is detected in the X-rays (Xue et al. 2011). Its faintness $\left(F_{\mathrm{X}}=8.2 \times 10^{-17} \mathrm{erg} \mathrm{s}^{-1} \mathrm{~cm}^{-2}, L_{\mathrm{X}}=4.3 \times 10^{41} \mathrm{erg} \mathrm{s}^{-1}\right)$ seems consistent with a starburst rather than an AGN (Ranalli et al. 2003).

ID.6 (Figure 13) is located $\sim 4^{\prime \prime}$ east of ID.3 and probably belongs to a common physical structure (together with other galaxies with a spectroscopic $z \approx 1.09$ ). It is detected in the $1 \mathrm{~mm}$ continuum, and its $\mathrm{CO}$ spectrum shows an $\sim 3 \sigma$ excess at the frequency of the expected $\mathrm{CO}(2-1)$ line. The $\mathrm{CO}(4-3)$ transition is also detected with similar significance, although the best Gaussian fit of the line suggests a velocity shift of $\sim 200 \mathrm{~km} \mathrm{~s}^{-1}$ between the two transitions. This is most likely due to the poor $\mathrm{S} / \mathrm{N}$ of the two lines.

ID.7 (Figure 14) appears as a very compact source $\left(R_{\mathrm{e}}=0.7\right.$ $\mathrm{kpc})$ at $z=1.221$. Its Chandra image reveals the presence of a bright AGN $\left(F_{X}=1.01 \times 10^{-14} \mathrm{erg} \mathrm{s}^{-1} \mathrm{~cm}^{-2}\right.$ in Lehmer et al. (2005); $8.3 \times 10^{-15} \mathrm{erg} \mathrm{s}^{-1} \mathrm{~cm}^{-2}$ in Evans et al. (2010); $6.3 \times 10^{-15} \mathrm{erg} \mathrm{s}^{-1} \mathrm{~cm}^{-2}$ in Xue et al. 2011), yielding an $\mathrm{X}$-ray luminosity of $L_{\mathrm{X}}=5.4 \times 10^{43} \mathrm{erg} \mathrm{s}^{-1}$ ). It is not detected in the $1 \mathrm{~mm}$ dust continuum. A $3 \sigma$ excess is measured at the expected frequency of the $\mathrm{CO}(2-1)$ transition.

$I D .9$ and $I D .10$ (Figure 15) are both at $z \sim 2.3$. They are among the faintest galaxies in our sample in terms of $L_{\mathrm{IR}}$, just above the $10^{11} L_{\odot}$ cut. ID.9 appears as a compact bulge. ID.10 appears as a spiral galaxy with disturbed morphology. ASPECS data cover three $\mathrm{CO}$ transitions in these galaxies: $\mathrm{CO}(3-2)$, $\mathrm{CO}(7-6)$, and $\mathrm{CO}(8-7)$. None of these lines are detected.

ID.11 (Figure 16) is a compact $\left(R_{\mathrm{e}}=1.2 \mathrm{kpc}\right)$ galaxy at $z=0.895$. As for ID.8, the lowest- $J$ CO transition in the ASPECS coverage is the $\mathrm{CO}(4-3)$, which remains undetected.

\section{CO-BASED MEASUREMENTS}

\subsection{CO Luminosities and Associated $\mathrm{H}_{2}$ Masses}

We measure the line fluxes (or place limits) for all the $\mathrm{CO}$ transitions covered in both the 3 and $1 \mathrm{~mm}$ line scans. We extract the CO spectra at the position of the optical coordinates of the sources in our sample. We fit the lowest- $J$ transitions accessible with ASPECS data with a Gaussian profile; in the case of a detection, we fit the higher- $J$ lines imposing the same line width. We consider as detections those cases where the flux obtained in the Gaussian fit is $>3 \times$ its uncertainty. If the line is not detected, we assume a fiducial line width of $300 \mathrm{~km} \mathrm{~s}^{-1}$, and we use the upper boundary of the $3 \sigma$ confidence range on the flux as upper limit. Table 2 reports the CO line fluxes, shifts compared with the nominal redshift, and the line width. The detected sources in our sample have a median CO flux of $0.19 \mathrm{Jy} \mathrm{km} \mathrm{s}^{-1}$ (considering only the lowest- $J$ transition observed in each object). For a comparison, the detected main-sequence galaxies in Tacconi et al. (2013) have a median $\mathrm{CO}$ flux of $0.57 \mathrm{Jy} \mathrm{km} \mathrm{s}^{-1}$, i.e., $3 \times$ higher than the median flux of our detections.
Table 2

CO Lines in the Galaxies of our Sample

\begin{tabular}{|c|c|c|c|c|}
\hline (1) & $\begin{array}{l}J_{\text {up }} \\
(2)\end{array}$ & $\begin{array}{c}\Delta v \\
\left(\mathrm{~km} \mathrm{~s}^{-1}\right) \\
(3)\end{array}$ & $\begin{array}{r}F_{\text {line }} \\
\left(\mathrm{Jy} \mathrm{km} \mathrm{s}^{-1}\right) \\
(4)\end{array}$ & $\begin{array}{c}\text { FWHM } \\
\left(\mathrm{km} \mathrm{s}^{-1}\right) \\
(5)\end{array}$ \\
\hline 1 & 3 & $-45 \pm 8$ & $0.723_{-0.003}^{+0.003}$ & $504 \pm 12$ \\
\hline 1 & 7 & $-150 \pm 120$ & $0.786_{-0.006}^{+0.006}$ & $504^{\mathrm{a}}$ \\
\hline 1 & 8 & $-45 \pm 70$ & $1.098_{-0.005}^{+0.005}$ & $504^{\mathrm{a}}$ \\
\hline 2 & 2 & $135 \pm 9$ & $0.443_{-0.007}^{+0.007}$ & $538 \pm 13$ \\
\hline 2 & 5 & $135 \pm 45$ & $0.502_{-0.090}^{+0.090}$ & $538^{\mathrm{a}}$ \\
\hline 2 & 6 & $-45 \pm 45$ & $0.820_{-0.100}^{+0.100}$ & $538^{\mathrm{a}}$ \\
\hline 3 & 2 & $-37 \pm 8$ & $0.135_{-0.003}^{+0.003}$ & $57 \pm 12$ \\
\hline 3 & 5 & $\ldots$ & $<0.021$ & $57^{\mathrm{a}}$ \\
\hline 4 & 2 & $52 \pm 7$ & $0.180_{-0.006}^{+0.006}$ & $82 \pm 11$ \\
\hline 4 & 4 & $\ldots$ & $<0.121$ & $82^{\mathrm{a}}$ \\
\hline 5 & 2 & $220 \pm 35$ & $0.190_{-0.040}^{+0.040}$ & $352 \pm 11$ \\
\hline 5 & 4 & $-28 \pm 40$ & $0.390_{-0.065}^{+0.065}$ & $352^{\mathrm{a}}$ \\
\hline 6 & 2 & $-160 \pm 70$ & $0.340_{-0.070}^{+0.060}$ & $530 \pm 11$ \\
\hline 6 & 4 & $230 \pm 70$ & $0.370_{-0.090}^{+0.090}$ & $182^{\mathrm{a}}$ \\
\hline 7 & 2 & $150 \pm 17$ & $0.104_{-0.029}^{+0.019}$ & $150 \pm 11$ \\
\hline 7 & 5 & $\ldots$ & $<0.106$ & $150^{\mathrm{a}}$ \\
\hline 8 & 4 & $\ldots$ & $<0.059$ & $\ldots$ \\
\hline 9 & 3 & $\cdots$ & $<0.076$ & $\ldots$ \\
\hline 9 & 7 & $\ldots$ & $<0.012$ & $\ldots$ \\
\hline 9 & 8 & $\ldots$ & $<0.230$ & $\ldots$ \\
\hline 10 & 3 & $\ldots$ & $<0.048$ & $\ldots$ \\
\hline 10 & 6 & $\ldots$ & $<0.144$ & $\ldots$ \\
\hline 10 & 7 & $\cdots$ & $<0.465$ & $\ldots$ \\
\hline 11 & 4 & $\ldots$ & $<0.015$ & $\ldots$ \\
\hline
\end{tabular}

Note. (1) Source ID. (2) Upper $J$ of the CO transition. (3) Velocity shift, compared with the redshift quoted in Table 1. (4) Line flux. (5) Line width, expressed as the full width at half maximum (FWHM) from the Gaussian fit. ${ }^{\text {a }}$ Fixed from the fit of a lower $J$ line.

The luminosity of the lowest- $J$ transitions observed in our molecular scans is transformed into the equivalent ground-state luminosity $L_{\mathrm{CO}(1-0)}^{\prime}$ using $L_{\mathrm{CO}(J-[J-1])}^{\prime} / r_{J 1}$, where we adopt the recent $\mathrm{CO}$ excitation ladder of main-sequence galaxies derived by Daddi et al. (2015): $r_{21}=0.76 \pm 0.09$, $r_{31}=0.42 \pm 0.07$, and $r_{41}=0.31 \pm 0.06 .{ }^{30}$ The uncertainty in $L_{\mathrm{CO}(1-0)}^{\prime}$ accounts for both the measured flux uncertainty and the standard deviation in the $r_{J 1}$ values in the sample studied by Daddi et al. (2015). The molecular gas masses are then derived as

$$
\frac{M_{\mathrm{H} 2}}{M_{\odot}}=\frac{\alpha_{\mathrm{CO}}}{r_{J 1}} \frac{L_{(J-[J-1])}^{\prime}}{\mathrm{K} \mathrm{km} \mathrm{s}^{-1} \mathrm{pc}^{2}} .
$$

We adopt $\alpha_{\mathrm{CO}}=3.6 M_{\odot}\left(\mathrm{K} \mathrm{km} \mathrm{s}^{-1} \mathrm{pc}^{2}\right)^{-1}$ (Daddi et al. 2010b). This conversion factor has been demonstrated to be appropriate for main-sequence galaxies through comparisons with dynamical masses (Daddi et al. 2010b), CO line SEDfitting (Daddi et al. 2015), and detailed dust-SED modeling (Genzel et al. 2015). In Section 5.5 we further discuss the implications of our $\alpha_{\mathrm{CO}}$ assumption. Table 3 lists the values of molecular gas masses that we derive for each source. We then combine these measurements or limits on the molecular gas mass with properties of the galaxies inferred from the SED

\footnotetext{
${ }^{30}$ Daddi et al. (2015) do not measure $\mathrm{CO}(4-3)$ in the galaxies in their sample. The value of $r_{41}$ adopted here is extrapolated from their measurements of $r_{31}$ and $r_{51}$, in the case of a CO ladder that peaks around $J \approx 5$ (see their Figure 10, left). As uncertainty, we adopt a conservative $20 \%$ error.
} 
Table 3

CO Luminosities and CO-based Galaxy Parameters

\begin{tabular}{|c|c|c|c|c|c|c|c|}
\hline (1) & $\begin{array}{l}z \\
(2)\end{array}$ & $\begin{array}{l}J_{\text {up }} \\
\text { (3) }\end{array}$ & $\begin{array}{c}L^{\prime} \\
\left(\times 10^{9} \mathrm{Km} \mathrm{s}^{-1} \mathrm{pc}^{2}\right)\end{array}$ & $\begin{array}{c}L_{\mathrm{CO}(1-0)}^{\prime} \\
\left(\times 10^{9} \mathrm{~K} \mathrm{~km} \mathrm{~s}^{-1} \mathrm{pc}^{2}\right) \\
(5)\end{array}$ & $\begin{array}{c}M_{\mathrm{H} 2} \\
\left(\times 10^{9} M_{\odot}\right) \\
\quad(6)\end{array}$ & $\begin{array}{c}M_{\mathrm{H} 2} / M_{*} \\
\text { (7) }\end{array}$ & $\begin{array}{l}t_{\text {depl }} \\
(\mathrm{Gyr}) \\
(8)\end{array}$ \\
\hline 1 & 2.543 & 3 & $24.03_{-0.10}^{+0.10}$ & $57_{-10}^{+10}$ & $206_{-34}^{+34}$ & $12_{-2}^{+2}$ & $3.3_{-0.6}^{+0.7}$ \\
\hline 2 & 1.551 & 2 & $13.71_{-0.27}^{+0.21}$ & $18_{-2}^{+2}$ & $65_{-8}^{+8}$ & $0.24_{-0.05}^{+0.05}$ & $0.9_{-0.4}^{+0.6}$ \\
\hline 3 & 1.382 & 2 & $3.364_{-0.08}^{+0.07}$ & $4.4_{-0.5}^{+0.5}$ & $15.9_{-1.9}^{+1.9}$ & $0.30_{-0.07}^{+0.08}$ & $0.9_{-0.3}^{+0.6}$ \\
\hline 4 & 1.088 & 2 & $2.831_{-0.09}^{+0.09}$ & $3.7_{-0.5}^{+0.5}$ & $13.4_{-1.7}^{+1.7}$ & $0.48_{-0.11}^{+0.13}$ & $0.6_{-0.3}^{+0.4}$ \\
\hline 5 & 1.098 & 2 & $3.089_{-0.66}^{+0.70}$ & $4.1_{-1.0}^{+1.0}$ & $15_{-4}^{+4}$ & $2.5_{-0.6}^{+0.7}$ & $0.33_{-0.09}^{+0.09}$ \\
\hline 6 & 1.094 & 2 & $5.388_{-1.16}^{+0.91}$ & $7.1_{-1.7}^{+1.5}$ & $25_{-6}^{+5}$ & $0.34_{-0.09}^{+0.10}$ & $1.6_{-0.7}^{+0.9}$ \\
\hline 7 & 1.221 & 2 & $2.047_{-0.57}^{+0.37}$ & $2.7_{-0.8}^{+0.6}$ & $10_{-3}^{+2}$ & $0.6_{-0.2}^{+0.16}$ & $0.066_{-0.020}^{+0.016}$ \\
\hline 8 & 0.999 & 4 & $<0.20$ & $<0.63$ & $<2.3$ & $<0.03$ & $<0.06$ \\
\hline 9 & 2.447 & 3 & $<2.4$ & $<5.6$ & $<21$ & $<8$ & $<1.8$ \\
\hline 10 & 2.224 & 3 & $<2.2$ & $<5.3$ & $<19$ & $<1.6$ & $<0.9$ \\
\hline 11 & 0.895 & 4 & $<0.53$ & $<1.7$ & $<6.2$ & $<0.4$ & $<0.15$ \\
\hline
\end{tabular}

Notes. (1) Source ID. (2) Redshift. (3) Observed transition. (4) Line luminosity. (5) Equivalent CO(1-0) luminosity, assuming the $r_{J 1}$ ratios in Daddi et al. (2015). (6) Molecular gas mass, assuming $\alpha_{\mathrm{CO}}=3.6 M_{\odot}\left(\mathrm{K} \mathrm{km} \mathrm{s}^{-1} \mathrm{pc}^{2}\right)^{-1}$. (7) Molecular-to-stellar mass ratio, $M_{\mathrm{H} 2} / M_{*}$. (8) Depletion time, $t_{\mathrm{dep}}=M_{\mathrm{H} 2} / \mathrm{SFR}$.
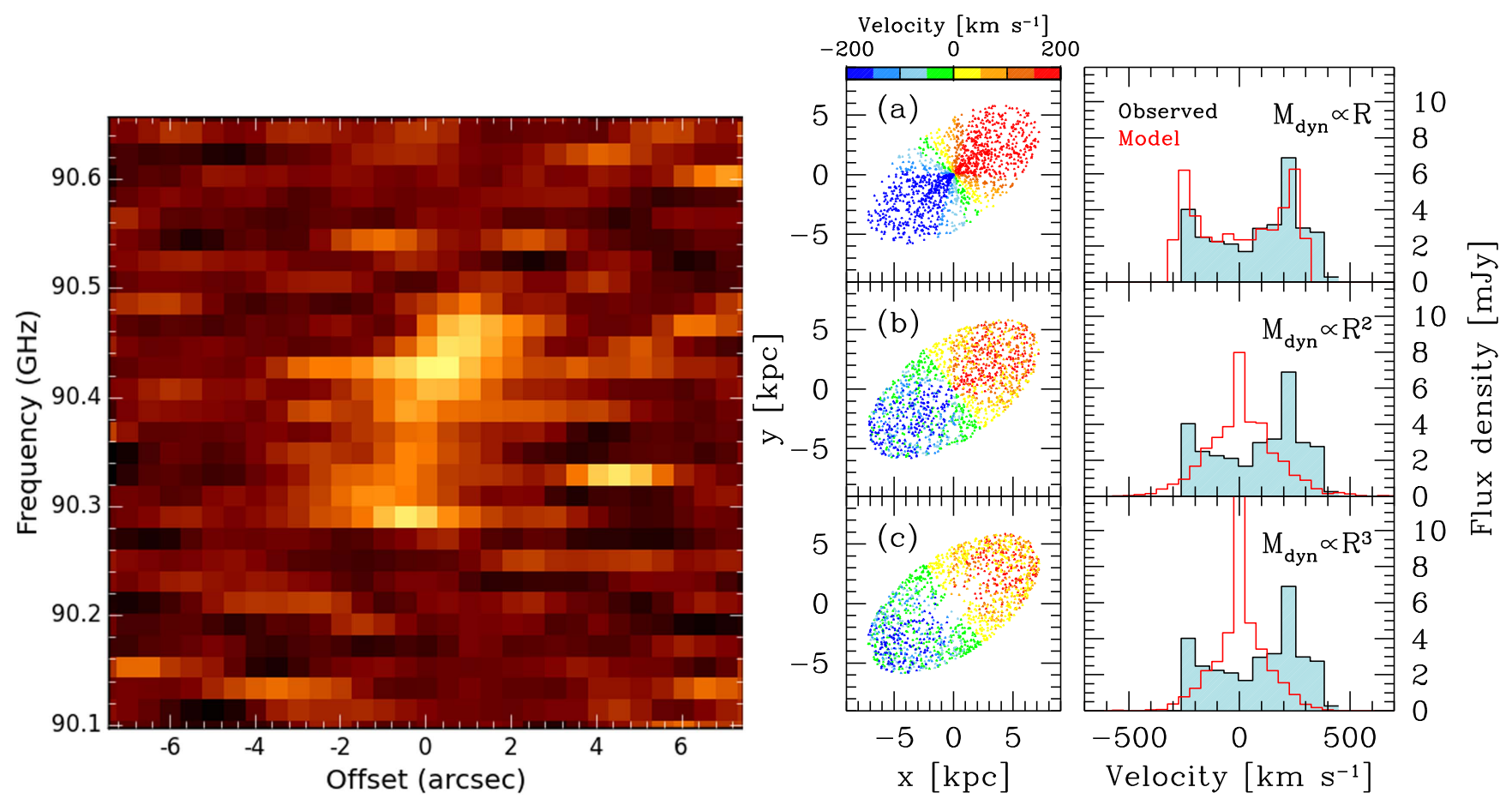

Figure 4. Left panel: position-velocity diagram of the $\mathrm{CO}(2-1)$ emission in ID.2, extracted along the major axis of the galaxy. A velocity gradient is apparent. Right panel: simulated velocity maps of ID.2, assuming that the gas is emitted in a disk geometry and that the $\operatorname{CO}(2-1)$ emission traces the mass distribution. The three models refer to different radial scalings of the dynamical mass: (a) $M_{\mathrm{dyn}} \propto R$ (thus $v_{\text {rot }}=$ const); (b) $M_{\mathrm{dyn}} \propto R^{2}$ (i.e., constant surface mass density in the disk; $v_{\text {rot }} \propto \sqrt{R}$ ); (c) $M_{\text {dyn }} \propto R^{3}$ (i.e., constant volume mass density; $v_{\text {rot }} \propto R$, i.e., solid rotator). All models assume a dynamical mass $M_{\text {dyn }}=2 \times 10^{11} M_{\odot}$ at $R=8.3 \mathrm{kpc}$. The expected line profiles (red histograms) are compared with the observed one (black dots). The flat rotation curve model seems to best reproduce the observed line profile.

fitting (in particular, the stellar mass $M_{*}$ and the SFR) to compute the molecular-to-stellar mass ratio $M_{\mathrm{H} 2} / M_{*}$ and the depletion timescale $t_{\mathrm{dep}}=M_{\mathrm{H} 2} / \mathrm{SFR}$ (see Table 3).

\subsection{Size of the CO-emitting Region in ID.2}

In the case of ID.2, our ALMA observations spatially resolve the $\mathrm{CO}(2-1)$ emission over $>15 \mathrm{kpc}$, despite the relatively coarse spatial resolution of the $3 \mathrm{~mm}$ data. A clear velocity gradient is observed in the line emission, as shown in Figure 4. While the resolution and the signal-to-noise ratio are too poor for an accurate modeling of the gas dynamics, we obtain an estimate of the dynamical mass assuming that the gas is rotating in a disk with the inclination derived from the HST near-IR imaging $\left(\mathrm{PA}=-55^{\circ}\right.$, inclination $=60^{\circ}$ with respect to the line of sight). We then assume a radial distribution of the mass that scales as $M_{\text {dyn }} \propto R^{\gamma}$, where $\gamma=1$ yields the flat rotation curves typically observed in galaxies; $\gamma=2$ implies a constant surface density of mass in the disk, and yields $v_{\text {rot }} \propto \sqrt{R}$; and $\gamma=3$ corresponds to a solid rotator $\left(v_{\text {rot }} \propto R\right)$. We then generated mock velocity maps for these three cases, assuming that the $\mathrm{CO}$ light traces the mass distribution; and we 
inferred expected line profiles (see Figure 4). The $\gamma=1$ case shows the typical "double-horned" profile observed in local spiral galaxies. This seems to provide a better description of the observed $\mathrm{CO}(2-1)$ line than the other two models, which fail to reproduce the extension of the blue wing of the line. The implied dynamical mass is $M_{\text {dyn }} \approx 2 \times 10^{11} M_{\odot}$ at $R=8.3 \mathrm{kpc}$ (= the effective radius). We stress, however, that this estimate is highly dependent on the model assumptions. A firmer estimate of the dynamical mass in this galaxy requires deeper data at higher spatial resolution.

ID. 2 also appears in the SINFONI Integral field spectroscopy survey in the near-IR (SINS; Förster Schreiber et al. 2009) as GMASS-1084 (see also Kurk et al. 2013). SINS investigated the morphology and kinematics of ionized gas (as traced by the $\mathrm{H} \alpha$ hydrogen line) in a sample of galaxies at $z=1-3.5$. The $\mathrm{H} \alpha$ line in ID.2 is emitted on a smaller region (half-light radius $R_{1 / 2}=3.1 \pm 1.0 \mathrm{kpc}$ ) than the CO. The observed $\mathrm{H} \alpha$ circular velocity is $67 \pm 9 \mathrm{~km} \mathrm{~s}^{-1}$, which is corrected into $230 \mathrm{~km} \mathrm{~s}^{-1}$ by assuming a low-inclination angle $\left(\sim 20^{\circ}\right)$. This yields a dynamical mass of $1.2 \times 10^{11} M_{\odot}$, roughly consistent with our estimate, especially if one considers that the high level of dust reddening ( $A_{V}=2.4 \mathrm{mag}$ from our global MAGPHYS fit) in this source may be responsible for suppressing $\mathrm{H} \alpha$ in parts of this galaxy. We note, however, that the SED fit of this source in Förster Schreiber et al. (2009) yields a stellar mass of only $M_{*}=3.61_{-0.60}^{+0.34} \times 10^{10} M_{\odot}$ and a high SFR $=490_{-31}^{+190} M_{\odot} \mathrm{yr}^{-1}$ (i.e., $L_{\mathrm{IR}} \approx 5.7 \times 10^{12} L_{\odot}$ ). This last estimate disagrees with our dust continuum measurements: e.g., assuming a modified blackbody template with $\beta=1.6$ and $T_{\text {dust }}=25 \mathrm{~K}$, such a high SFR would imply a dust-continuum flux density of $11 \mathrm{mJy}$ at $1.2 \mathrm{~mm}$ (observed: $0.22 \pm 0.02 \mathrm{mJy}$ ) and of $32 \mathrm{mJy}$ at $160 \mu \mathrm{m}$ (observed: $6.9 \pm 0.3 \mathrm{mJy}$ ).

As seen from Figure 4, the molecular gas, as traced through $\mathrm{CO}$ emission, is extended on scales of $>15 \mathrm{kpc}\left(>2^{\prime \prime}\right.$ at $z=1.552$ ), i.e., comparable to that of the stellar disk. On the other hand, the $1 \mathrm{~mm}$ dust continuum is unresolved at $1.5^{\prime \prime} \times 1.0^{\prime \prime}$ resolution, i.e., it is significantly smaller than that of the CO (see Figure 11). This is not an effect of interferometric filtering or sensitivity of the $1 \mathrm{~mm}$ data. When we convolve the $1 \mathrm{~mm}$ continuum data to the synthesized beam of the $3 \mathrm{~mm}$ data, we do not recover the size seen in $\mathrm{CO}$ emission. This serves as a cautionary note that $\mathrm{CO}$ and dust sizes may not be the same. As a consequence, the masses deduced from these measurements may trace different regions or components in the galaxy (for other examples of a mismatch between $\mathrm{CO}$ and dust morphology in high-redshift galaxies, see Riechers et al. 2011; Hodge et al. 2015; Spilker et al. 2015). This may explain some of the differences between gas mass estimates derived from $\mathrm{CO}$ and dust imaging, with the gas masses derived from dust emission being typically lower than those derived from $\mathrm{CO}$ (see Section 5.5): At the observed wavelength $(1.2 \mathrm{~mm})$, dust is optically thin (with the only exception of ID.1, all the sources in our sample globally have $\Sigma_{\text {gas }} \ll 10^{4} M_{\odot} \mathrm{pc}^{-2}$, i.e., $N_{\mathrm{H} 2} \ll 10^{24} \mathrm{~cm}^{-2}$; this yields $\tau$ $[242 \mathrm{GHz}] \ll 0.1$ for solar metallicities, adopting the Draine \& Lee 1984 formalism). The CO low-J emission, on the other hand, is optically thick practically everywhere in galaxies.

\subsection{CO Excitation}

As shown in Table 1, ASPECS covers 2-3 different CO transitions in 9 out of 11 galaxies in our sample. Figure 5 shows the inferred constraints on the $\mathrm{CO}$ excitation ladder. In

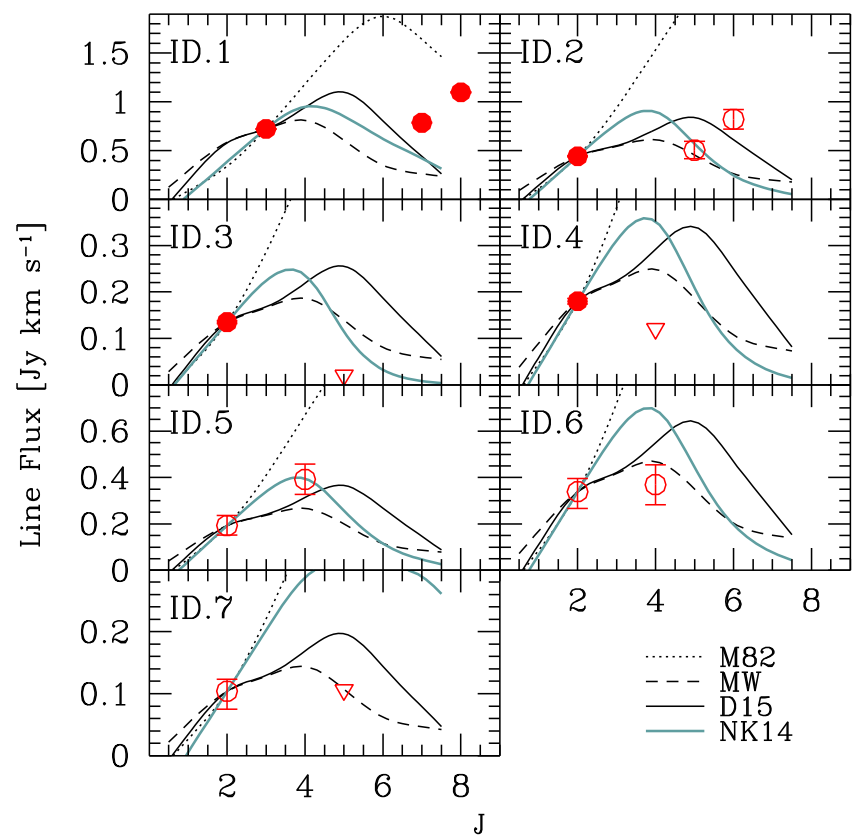

Figure 5. CO ladder for the galaxies of our sample detected in CO. Filled symbols mark the transitions detected in our blind search (see Paper I), while empty symbols mark lines that do not match the blind detection requirements. Upper limits, marked with triangles, correspond to $3 \sigma$ limits. The excitation templates of the Milky Way and M82 are taken from Weiß et al. (2007), while the main-sequence galaxy template is from Daddi et al. (2015) (D15). Finally, the theoretical predictions based on the SFR surface density are based on Narayanan \& Krumholz (2014) (NK14). All templates are scaled to match the observed CO flux of the lowest $J$ transition detected in ASPECS. The galaxies in our sample typically show a modest to very low $\mathrm{CO}$ excitation. ID.1 (= ASPECS $3 \mathrm{~mm} .1,1 \mathrm{~mm} .1,2$ ) and ID.5 show slightly higher CO excitation than the template by Daddi et al. (2015), although still well below the highexcitation case of the M82 starburst template.

ID.1, all three observed transitions $[\mathrm{CO}(3-2), \mathrm{CO}(7-6)$, and $\mathrm{CO}(8-7)]$ were detected in our blind search for line emission (ASPECS $3 \mathrm{~mm} .1,1 \mathrm{~mm} .1$, and $1 \mathrm{~mm} .2$, respectively). In ID.2, the $\mathrm{CO}(2-1)$ line appears in the results of our blind search (ASPECS $3 \mathrm{~mm} .2$ ). The $\mathrm{CO}(5-4)$ and $\mathrm{CO}(6-5)$ lines are also observed, but because of their lower significance, they were not detected in our blind search. In particular, the $\mathrm{CO}(6-5)$ line is very noisy, as it is found at the high-frequency end of the $1 \mathrm{~mm}$ spectral scan, and it is spatially located at the edge of our mosaic. The $\mathrm{CO}(2-1)$ transitions in ID. 3 and ID. 4 are also identified in our blind search (ASPECS $3 \mathrm{~mm} .3$ and $3 \mathrm{~mm} .5$, respectively). However, the $\mathrm{CO}(5-4)$ line in ID.3 and the $\mathrm{CO}(4-3)$ line in ID.4 are not detected. In particular for ID.3, this places very strong limits on the $\mathrm{CO}$ excitation of this galaxy, significantly below the average CO ladder of the Milky Way disk (see Figure 5). In ID.5 and ID.6, we detect both $\mathrm{CO}(2-1)$ and $\mathrm{CO}(4-3)$. Finally, in ID.7 we only have a tentative detection of $\mathrm{CO}(2-1)$, while the $\mathrm{CO}(5-4)$ transition remains undetected. No other line is detected in the remainder of our sample.

In Figure 5 we compare our measurements and limits with the $\mathrm{CO}$ excitation templates of the Milky Way disk, and of the starburst in M82 (Weiß et al. 2007). Additionally, we compare with the average template for high- $z$ main-sequence galaxies by Daddi et al. (2015) and with the theoretical predictions based on the SFR surface density by Narayanan \& Krumholz (2014) (see Table 1 and the discussion in Section 5.2.2). In no case do we find starburst-like $\mathrm{CO}$ excitation that would be comparable with 
the center of M82 (Weiß et al. 2007) or with what is typically observed in high- $z$ sub-mm galaxies (SMGs; e.g., Bothwell et al. 2013; Spilker et al. 2014). ID.1 shows a CO(7-6)/CO(3-2) ratio $r_{73}=0.2$, consistent with a high-density photon-dominated region (Meijerink et al. 2007). On the other hand, the $\mathrm{CO}(8-7)$ transition appears brighter, implying that a high-excitation component of the interstellar medium (ISM) might be in place. Interestingly, ID. 2 shows a lower excitation (in particular in the $\mathrm{CO}[5-4] / \mathrm{CO}[2-1]$ ratio, which is consistent with Milky Way excitation). This difference in $\mathrm{CO}$ excitation is remarkable when we consider that ID. 1 is not detected with Chandra $\left(L_{\mathrm{X}}<6 \times 10^{42} \mathrm{erg} \mathrm{s}^{-1}\right)$, whereas ID. 2 shows a bright X-ray detection, indicative of the presence of a central AGN. The $\mathrm{X}$-ray emission from the AGN can boost the emission of high-J $\mathrm{CO}$ transitions. The $\mathrm{CO}(7-6) / \mathrm{CO}(3-2)$ ratio $r_{73}$ is typically $0.16-0.63$ in high-density photon-dominated regions that are powered by star formation (as in ID.2), but it can reach values as high as $r_{73}=30$ in the presence of intense X-ray illumination (Meijerink et al. 2007). This might explain the higher CO excitation observed in high- $z$ QSOs with respect to submillimeter galaxies (Carilli \& Walter 2013). The lack of such highexcitation feature suggests that the central AGN activity in ID.2 has no major impact on its global $\mathrm{CO}$ properties. We attribute the higher excitation in ID.1 to the much more compact emission in this galaxy. As shown in Table 1, ID.1 has a radius that is only $\sim 1 / 5$ of that of ID.2, which translates into a difference in surface area of $\sim 24$. Our MAGPHYS-based SFR estimates are comparable $\left(\sim 70 M_{\odot} \mathrm{yr}^{-1}\right)$, thus the surface density of star formation $\left(\Sigma_{\mathrm{SFR}}\right)$ is much higher in ID.1. This is also discussed in Section 5.2 below. The increased radiation field intensity caused by the high SFR surface density and/or the higher gas density are very likely the reason for the increased $\mathrm{CO}$ excitation (see Narayanan \& Krumholz 2014).

\section{DISCUSSION}

In the following we discuss the sources of our sample in the broad context of gas properties in high-redshift galaxies.

\subsection{Location in the Galaxy "Main-sequence" Plot}

The stellar masses of the galaxies in our sample range between $(2.8-275) \times 10^{9} M_{\odot}$ (two orders of magnitude). The $L_{\mathrm{IR}}>10^{11} L_{\odot}$ cut in our sample definition selects sources with $\mathrm{SFR}>10 M_{\odot} \mathrm{yr}^{-1}$. The measured SFRs range between $12-150 M_{\odot} \mathrm{yr}^{-1} .31$

Figure 1 shows the location of our galaxies in the $M_{*}-\mathrm{SFR}$ ("main sequence") plane. We plot all the galaxies in the field with an F850LP or F160W magnitude brighter than 27.5 mag (this cut allows us to remove sources with highly uncertain SED fits). The galaxies in the present sample are highlighted with large symbols. The different redshifts of the sources are indicated by different colors. As expected from the known evolution of the "main sequence" of star-forming galaxies (e.g.,

\footnotetext{
31 We note that the FAST analysis by Skelton et al. (2014) yields consistent SFRs for ID.1, ID.3, and ID.10, but different values (by a factor $2 \times$ or more) for ID. $2 \quad\left(6 M_{\odot} \mathrm{yr}^{-1}\right), \quad$ ID.4 $\quad\left(50 M_{\odot} \mathrm{yr}^{-1}\right), \quad$ ID.5 $\left(21 M_{\odot} \mathrm{yr}^{-1}\right), \quad$ ID.6 $\left(3.7 M_{\odot} \mathrm{yr}^{-1}\right), \quad$ ID.7 $\quad\left(230 M_{\odot} \mathrm{yr}^{-1}\right), \quad$ ID.8 $\left(0.01 M_{\odot} \mathrm{yr}^{-1}\right), \quad$ and ID.11 $\left(2.6 M_{\odot} \mathrm{yr}^{-1}\right)$. No FAST-based SFR estimate is available for ID.9. These differences are most likely due to (1) different assumptions on the source redshifts; (2) different coverage of the SED photometry, in particular thanks to the addition of the $1 \mathrm{~mm}$ continuum constraint in our MAGPHYS analysis; (3) different working assumptions in the two codes. In particular, FAST relies on relatively limited prescriptions for the dust attenuation and star formation history, and does not model the dust emission.
}

Whitaker et al. 2012; Schreiber et al. 2015), sources at higher redshifts tend to have a higher SFR per unit stellar mass. Comparing with the Herschel-based results by Schreiber et al. (2015), we find that half of the galaxies in our sample (ID.1, 2, $4,8,9,10$ ) lie on the main sequence (within a factor $3 \times$ ) at their redshift. Three galaxies (ID.5, 7, 11) are above the main sequence (in the "starburst" region), and the remaining two galaxies (ID.3 and ID.6) show an SFR $\sim 3 \times$ lower than mainsequence galaxies at these redshifts and stellar masses. Similar conclusions are reached when we compare our results with the main-sequence fits by Whitaker et al. (2012) (see Figure 1).

\subsection{Star Formation Law}

The relationship between the total infrared luminosity $\left(L_{\mathrm{IR}}\right.$, a proxy for the SFR) and the total CO luminosity $\left(L_{\mathrm{CO}}^{\prime}\right.$, a proxy for the available gas mass) of galaxy samples is typically referred to as the "integrated Schmidt-Kennicutt" law (Schmidt 1959; Kennicutt 1998; Kennicutt \& Evans 2012), or, more generally, the "star formation" law. Sometimes average surface density values are derived from these quantities, resulting in average surface SFR densities $\left(\Sigma_{\mathrm{SFR}}\right)$ and gas densities $\left(\Sigma_{\mathrm{gas}}\right)$. We here explore both relations.

\subsubsection{Global Star Formation Law: IR Versus CO Luminosities}

In Figure 6 we compare the IR and $\mathrm{CO}(1-0)$ luminosities of our sources with respect to a compilation of galaxies both at low and high redshift from the review by Carilli \& Walter (2013), and with the secure blind detections in Decarli et al. (2014). For galaxies in our sample that are undetected in CO, we plot the corresponding $3 \sigma$ limit on the line luminosities. The IR-CO luminosity empirical relation motivates the $L_{\mathrm{IR}}$ cut in our sample selection, as galaxies with $L_{\mathrm{IR}}>10^{11} L_{\odot}$ should have $\mathrm{CO}$ emission brighter than $L^{\prime} \approx 3 \times 10^{9} \mathrm{~K} \mathrm{~km} \mathrm{~s}^{-1} \mathrm{pc}^{2}$ (i.e., our typical sensitivity limit in ASPECS; see Paper I). All the galaxies in our sample should therefore be detected in $\mathrm{CO}$. We find that most of the CO-detected galaxies in our sample lie along the one-to-one relation, followed by local spiral galaxies as well as color-selected main-sequence galaxies at $1<z<3$ (Daddi et al. 2010b; Genzel et al. 2010, 2015; Tacconi et al. 2013). Only two galaxies significantly deviate: ID.5, which appears on the upper envelope of the IR-CO relation, close to high-redshift starburst galaxies; and ID.7, which is largely underluminous in $\mathrm{CO}$ for its bright IR emission. As discussed in the previous section, these two galaxies appear as starbursts in Figure 1. Moreover, ID.7 hosts a bright AGN. If the AGN contamination at optical wavelengths is significant, then our MAGPHYS-based SFR estimate is likely in excess (since MAGPHYS would associate some of the AGN light at rest-frame optical and UV wavelengths to a young stellar population), thus explaining the large vertical offset of this galaxy with respect to the "star formation law" shown in Figure 6. Notably, out of the $4 \mathrm{CO}$ non-detections in our sample, ID.9 and ID.10 are still consistent with the relation, while ID.8 and ID.11 are not. These two galaxies are located at $z=0.999$ and $z=0.895$, respectively. The lowest $J$ transition sampled in our study is $\mathrm{CO}(4-3)$. Their non-detections might be explained if the excitation in these two sources were much lower than what we assumed to infer $L_{\mathrm{CO}(1-0)}^{\prime}\left(r_{41}=0.31\right.$; see Section 4.1).

The sources that are also detected in the blind search for $\mathrm{CO}$ (ID.1, 2, 3, 4) tend to lie on the lower "envelope" of the plot. 


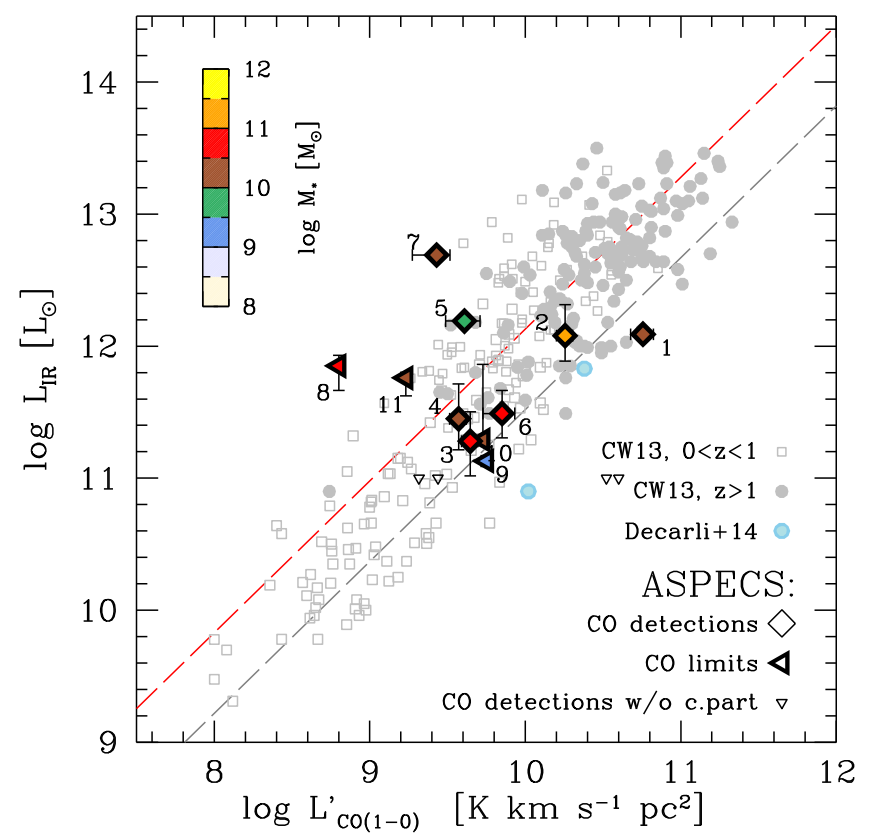

Figure 6. IR luminosity as a function of the $\mathrm{CO}(1-0)$ luminosity for both local galaxies (gray open symbols) and high-redshift sources $(z>1$, gray filled symbols) from the compilation in Carilli \& Walter (2013). The sources in our sample are shown with large symbols, using the same coding as in Figure 1. In addition, we also plot the $x$-axis position of the remaining $\mathrm{CO}$ lines found in our $3 \mathrm{~mm}$ blind search (downward triangles; see Paper I). The two parallel sequences of "normal" and "starburst" galaxies (Daddi et al. 2010b; Genzel et al. 2010) are shown as dashed lines (in gray and red, respectively). Our sources cover a wide range of luminosities, both in the $\mathrm{CO}$ line and in the IR continuum. Most of the sources in our sample lie along the sequence of "main sequence" galaxies. Four sources lie above the relation: ID.5, which still falls close to the high- $z$ starburst region; ID.7, in which the AGN contamination may lead to an excess of IR luminosity; and ID. 8 and ID.11, which are undetected in $\mathrm{CO}$, and which might be shifted toward the relation if we assumed very low $\mathrm{CO}$ excitation (as observed in other galaxies of our sample). Conversely, most of the sources detected in $\mathrm{CO}$ in our blind search (see Paper I) that lack an optical/IR counterpart lie significantly below the observed relation.

This is expected, as these galaxies have been selected based on their CO luminosity ( $x$-axis).

Figure 6 also shows the $x$-axis position of the remaining $\mathrm{CO}$ blind detections from the $3 \mathrm{~mm}$ search in Paper I. The CO luminosities of these lines are uncertain (the line identification is ambiguous in many cases, and a fraction of these lines is expected to be a false positive; see Paper I); however, it is interesting to note that these sources typically populate ranges of line luminosities that were previously unexplored at $z>1$ (see similar examples in Chapman et al. 2008, 2015b; Casey et al. 2011), and comparable with or even lower than the typical dust luminosities of local spiral galaxies. We emphasize that a significant fraction of these lines is expected to be real (see Paper I). Deeper data are required to better characterize these candidates.

\subsubsection{Average Surface Densities: SFR Versus Gas Mass}

We infer average estimates of $\Sigma_{\text {SFR }}$ and $\Sigma_{\text {gas }}$ by dividing the global SFR and $M_{\mathrm{H} 2}$ of the galaxy by a fiducial area set by the size of the stellar component, as $\mathrm{CO}$ and optical radii are typically comparable (Schruba et al. 2011; Tacconi et al. 2013). We thus use the information from the stellar morphology derived by van der Wel et al. (2012) and reported in Table 1 to infer $\Sigma_{\mathrm{SFR}}=\mathrm{SFR} /\left(2 \pi R_{e}^{2}\right)$ and $\Sigma_{\mathrm{H} 2}=M_{\mathrm{H} 2} /\left(2 \pi R_{e}^{2}\right)$, where

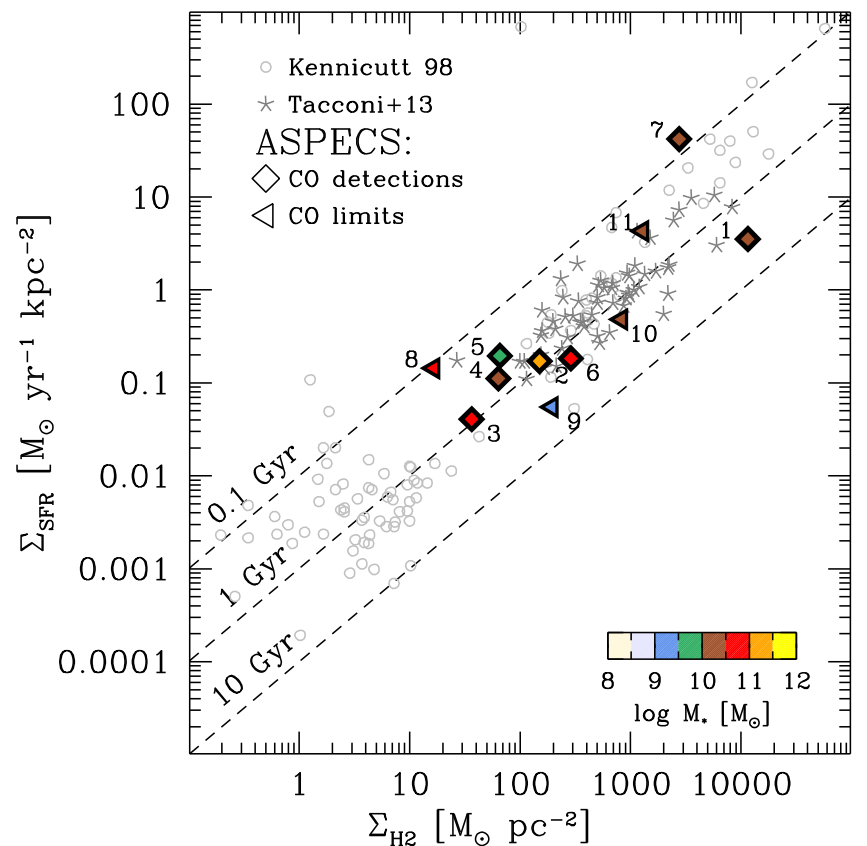

Figure 7. The "global" star formation law relates the average star formation rate surface density $\left(\Sigma_{\mathrm{SFR}}\right)$ with the average gas density in galaxies. Here we consider only the molecular gas phase $\left(\Sigma_{\mathrm{H} 2}\right)$. Each point in the plot refers to a different galaxy. We plot the reference samples from Kennicutt (1998) (corrected for the updated SFR calibration in Kennicutt \& Evans 2012), as well as the PHIBSS galaxies from Tacconi et al. (2013). Data from the literature have been corrected to match the same $\alpha_{\mathrm{CO}}=3.6 M_{\odot}\left(\mathrm{K} \mathrm{km} \mathrm{s}^{-1} \mathrm{pc}^{2}\right)^{-1}$ assumed in this work. The symbol code is the same as in Figure 6. The galaxies in our sample align along the $t_{\mathrm{depl}} \approx 1 \mathrm{Gyr}$, with the only exception of ID.7 and ID.8, which show a short depletion time. It is interesting to note that the two CO-brightest galaxies in our sample, ID.1 and ID.2, populate opposite extremes of the high- $z$ galaxy distribution, with the former being very compact (thus displaying higher SFR and gas densities), and the latter being very extended (thus showing lower SFR and gas densities).

$M_{\mathrm{H} 2}$ is our CO-based measurement of the molecular gas mass, and the factor 2 is due to the fact that the $R_{e}$ includes only half of the light of the galaxy (see a similar approach in Tacconi et al. 2013).

In Figure 7 we show the star formation law for average surface densities. Global measurements of local spiral galaxies and starbursts are taken from Kennicutt (1998), and corrected for the updated SFR calibration following Kennicutt \& Evans (2012) and to the $\alpha_{\mathrm{CO}}$ value adopted in this paper. We also plot the galaxies in the IRAM Plateau de Bure HIgh- $z$ Blue Sequence Survey (PHIBSS; Tacconi et al. 2013), again corrected to match the same $\alpha_{\mathrm{CO}}$ assumption used in this work, and the secure detections in Decarli et al. (2014). Interestingly, the two CO-brightest galaxies in our sample, ID.1 and ID.2, appear to populate opposite extremes of the density ranges observed in high- $z$ galaxies: ID. 1 appears very compact, thus reaching the top right corner of the plot $\left(\Sigma_{\text {gas }} \approx 10,000\right.$ $M_{\odot} \mathrm{pc}^{-2}$ ). On the other hand, in ID.2 the vast gas reservoir is spread over a large area (as apparent in Figure 4), thus yielding a globally low $\Sigma_{\text {gas }}$. We also find that most of the sources in our sample lie along the $t_{\mathrm{depl}} \approx 1 \mathrm{Gyr}$ line, in agreement with local spiral galaxies and the PHIBSS main-sequence galaxies. Only ID.7 and ID.8 lie closer to the $t_{\mathrm{depl}} \approx 0.1 \mathrm{Gyr}$ line. In particular, the offset of ID.7 with respect to the bulk of the sample in the context of the global star formation law (Figure 6) is combined here with the very compact size of the emitting region, thus isolating the source in the top left corner of the plot 


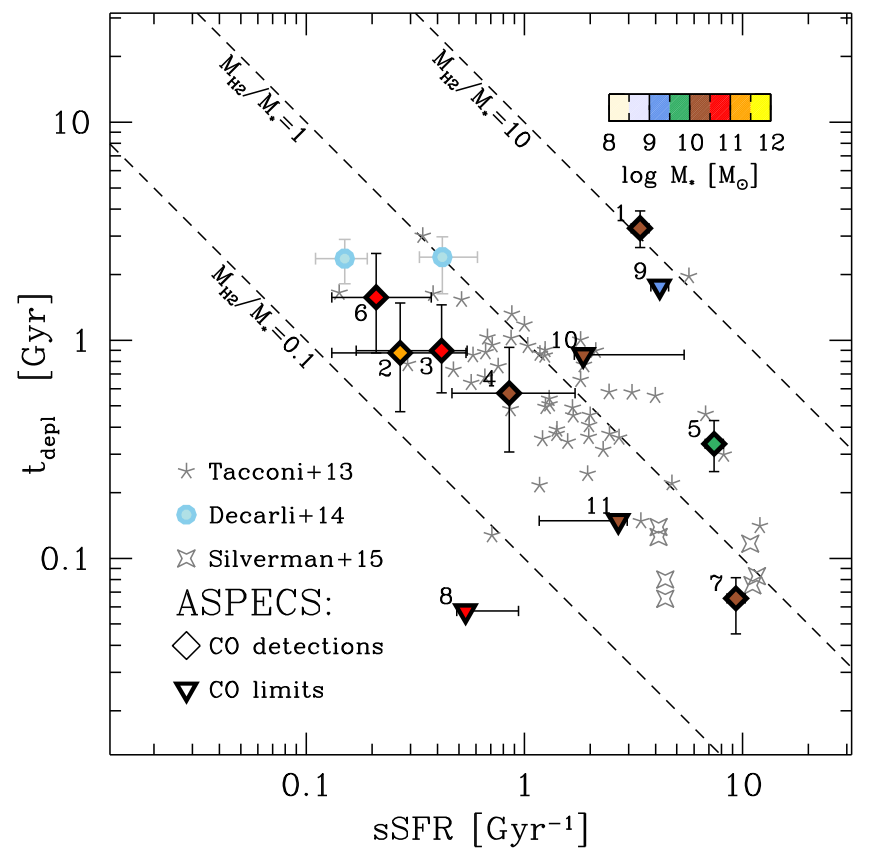

Figure 8. The depletion time $t_{\mathrm{depl}}=M_{\mathrm{H} 2} / \mathrm{SFR}$ as a function of the specific star formation rate $\mathrm{sSFR}=\mathrm{SFR} / M_{*}$ for the galaxies in our sample, the secure blind detections in Decarli et al. (2014), the PHIBSS sample by Tacconi et al. (2013), and the starburst sample in Silverman et al. (2015). The symbol code is the same as that in Figure 6. Starburst galaxies typically reside in the bottom right corner of the plot. Our ASPECS sources cover a wide range in parameter space, highlighting the diverse properties of these galaxies.

(see Figure 7). Once again, a significant AGN contamination in the estimates of both the rest-frame optical/UV luminosity and in the size of the emitting region could explain such an outlier. We also caution that, in some of these galaxies, optical and $\mathrm{CO}$ radii might differ.

\subsection{Depletion Times}

Figure 8 shows the depletion time, $t_{\mathrm{depl}}=M_{\mathrm{H} 2} / \mathrm{SFR}$, as a function of the specific SFR. This timescale sets how quickly the gas is depleted in a galaxy given the currently observed SFR (ignoring any gas repleneshing). Our data are compared again with the secure blind detections in Decarli et al. (2014), with the PHIBSS sample, and with the sample of starburst galaxies studied by Silverman et al. (2015) (in the latter case, we do not change the adopted value of $\alpha_{\mathrm{CO}}=1.1 M_{\odot}\left(\mathrm{K} \mathrm{km} \mathrm{s}^{-1} \mathrm{pc}^{2}\right)^{-1}$, as these are not mainsequence galaxies). Starburst galaxies tend to reside in the bottom right corner of the plot (they are highly star forming given their stellar mass, and they are using up their gaseous reservoir fast). Galaxies with large gas reservoirs and mild star formation populate the top left corner of the plot. Since the IR luminosity is proportional to the SFR and the CO luminosity is used to infer $M_{\mathrm{H} 2}$, the $y$-axis of this plot conceptually corresponds to a diagonal line (top left to bottom right) in Figure 6. In addition, the diagonal lines in Figure 8 mark the loci of the constant molecular-to-stellar mass ratio $M_{\mathrm{H} 2} / M_{*}$.

The sources in our sample range over almost 2 dex in sSFR and $t_{\text {depl }}$. Noticeably, ID. 1 is highly star forming (it resides slightly above the main sequence of star-forming galaxies at $z \sim 2.5$, see Figure 1), so we would expect it to reside in the bottom right corner of Figure 8; however, its gaseous reservoir is very large for its IR luminosity (see also Figure 6), thus

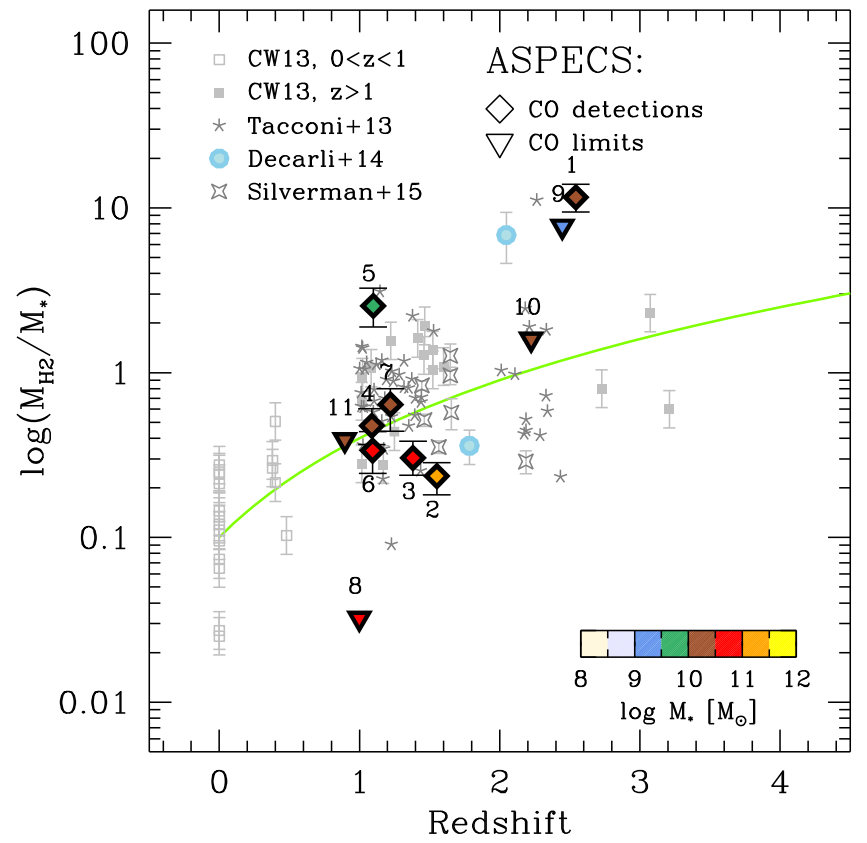

Figure 9. Gas mass fraction (defined as $M_{\mathrm{H} 2} / M_{*}$ ) as a function of redshift from various samples of galaxies in the literature (gray, from the compilation in Carilli \& Walter 2013), compared with the secure CO detections in Decarli et al. (2014), the PHIBSS sample (Tacconi et al. 2013), the starburst sample in Silverman et al. (2015), and our results from this work. The symbol coding is the same as in Figure 1. Our data seem to support the picture of a generally increasing $M_{\mathrm{H} 2} / M_{*}$ ratio in main-sequence galaxies as a function of redshift, as highlighted by the $f_{\text {gas }}=0.1 \times(1+z)^{2}$ green line (Geach et al. 2011; Magdis et al. 2012). In particular, ID.2 appears as a starburst with respect to its position above the "main sequence" in Figure 1, and shows a high $M_{\mathrm{H} 2} / M_{*}$ ratio. On the other hand, we also point out that significant upper limits are present (triangles).

placing ID. 1 in the top right corner of the plot $\left(M_{\mathrm{H} 2} / M_{*}=12\right)$. On the other hand, ID. 2 hosts an enormous reservoir of molecular gas, but because of its even higher stellar mass (yielding low sSFR), it resides on the left side of the plot $\left(M_{\mathrm{H} 2} / M_{*}=0.24\right)$. Their depletion timescales, however, are comparable (1-3 Gyr). We stress that these results are based on very high $\mathrm{S} / \mathrm{N} \mathrm{CO}$ line detections, and on very solid descriptions of the galaxy SEDs (see Figure 11). The sources that populate the starburst region in Figure 1 and reside in the top left part of Figure 6 (in particular, ID.5 and ID.7) consistently appear in the bottom right corner of Figure 8, that is, among starbursts.

\subsection{Gas-to-Stellar-Mass Ratios}

A useful parameter to investigate the molecular gas content in high- $z$ galaxies is the molecular gas to stellar mass ratio, $M_{\mathrm{H} 2} / M_{*}$. We prefer this parameter rather than the molecular gas fraction, $f_{\text {gas }}=M_{\mathrm{H} 2} /\left(M_{*}+M_{\mathrm{H} 2}\right)$, as the two involved quantities $\left(M_{\mathrm{H} 2}\right.$ and $\left.M_{*}\right)$ appear independently at the numerator and denominator of the fraction, so that the parameter is well defined even when we only have upper limits on $M_{\mathrm{H} 2}$. Figure 9 shows the dependence of $M_{\mathrm{H} 2} / M_{*}$ on redshift in the galaxies of our samples and in galaxies from the literature. This plot informs us of the typical gas content as a function of cosmic time, and can help us shed light on the origin of the cosmic star formation history (see, e.g., Geach et al. 2011; Magdis et al. 2012, and Paper III of this series). Color-selected starforming galaxies close to the epoch of galaxy assembly are 
Table 4

Gas Mass Estimates Based on the Dust Continuum

\begin{tabular}{|c|c|c|c|c|c|c|}
\hline (1) & $\begin{array}{l}z \\
\text { (2) }\end{array}$ & $\begin{array}{c}F_{\nu}(1.2 \mathrm{~mm}) \\
(\mu \mathrm{Jy}) \\
(3)\end{array}$ & $\begin{array}{c}k \text {-corr } \\
(4)\end{array}$ & $\begin{array}{c}\log M_{\text {gas, Groves }} \\
\left(M_{\odot}\right) \\
(5)\end{array}$ & $\begin{array}{c}\log M_{\mathrm{ISM}, \text { Scoville }} \\
\left(M_{\odot}\right) \\
(6)\end{array}$ & $\begin{array}{c}\log M_{\mathrm{gas}, \mathrm{MAGPHYS}} \\
\left(M_{\odot}\right) \\
(7)\end{array}$ \\
\hline 1 & 2.543 & $552.7 \pm 13.8$ & 0.374 & $11.02_{-0.011}^{+0.011}$ & $10.69_{-0.011}^{+0.011}$ & $10.53_{-0.17}^{+0.17}$ \\
\hline 2 & 1.551 & $223.1 \pm 21.6$ & 0.919 & $10.63_{-0.04}^{+0.04}$ & $10.33_{-0.04}^{+0.04}$ & $10.09_{-0.14}^{+0.13}$ \\
\hline 4 & 1.088 & $96.5 \pm 24.7$ & 1.665 & $10.24_{-0.10}^{+0.13}$ & $9.95_{-0.13}^{+0.10}$ & $9.78_{-0.20}^{+0.18}$ \\
\hline 5 & 1.098 & $46.4 \pm 14.9$ & 1.641 & $9.93_{-0.12}^{+0.17}$ & $9.63_{-0.17}^{+0.12}$ & $9.25_{-0.19}^{+0.14}$ \\
\hline
\end{tabular}

Note. Only sources detected at $1 \mathrm{~mm}$ in ASPECS are considered. (1) Source ID. (2) Redshift. (3) Observed 242, GHz $=1.2$, mm continuum flux density (see Paper II). (4) $k$ correction, expressed as the ratio between the flux density computed at $\lambda_{\text {restframe }}=500 \mu \mathrm{m}$ and the one at $\lambda_{\text {obs }}=1.2 \mathrm{~mm}$, assuming a modified blackbody template for the dust emission with $\beta=1.6$ and $T_{\text {dust }}=25 \mathrm{~K}$. (5) Gas mass based on the $1 \mathrm{~mm}$ flux density, derived following Equation (2) (Groves et al. 2015). (6) Gas mass based on the $1 \mathrm{~mm}$ flux density, derived following Equation (3) (Scoville et al. 2014, 2016). (7) Gas mass derived from the dust-mass estimate resulting from MAGPHYS SED fitting, assuming a dust-to-gas ratio DGR $=1 / 100$.

claimed to show large $M_{\mathrm{H} 2} / M_{*}$, with reservoirs of gas as large as (or even larger than) the stellar mass (i.e., $M_{\mathrm{H} 2} / M_{*} \sim 1$; see, e.g., Daddi et al. 2010a; Tacconi et al. 2010, 2013). Indeed, we find examples of very high gas fractions: ID.1 $\left(M_{\mathrm{H} 2} / M_{*}=12\right)$ and the starburst galaxy ID.5 $\left(M_{\mathrm{H} 2} / M_{*}=2.5\right)$ are the most extreme cases. However, it is interesting to note that we also find galaxies with very modest gas fractions, such as ID.2 $\left(M_{\mathrm{H} 2} / M_{*}=0.24\right)$. The CO-detected galaxies at $1.0<z<1.7$ in our sample show an average $M_{\mathrm{H} 2} / M_{*}$ ratio that is $\sim 2 \times$ lower than the average value for the PHIBSS sample at the same redshift, and closer to the global trend established in Geach et al. (2011) and Magdis et al. (2012). The non-detection of $\mathrm{CO}$ in ID.8 places particularly strict limits $\left(M_{\mathrm{H} 2} / M_{*}<\right.$ 0.03 ). If the lack of detection is attributed to the very low $\mathrm{CO}$ excitation in this galaxy, then it would take a $10 \times$ lower $r_{41}$ (i.e., $r_{41} \approx 0.03$ ) to shift ID.8 on the average trend reported by Geach et al. (2011).

\subsection{CO versus Dust-based ISM Masses}

In addition to the $\mathrm{CO}$ line measurements, 6 of the 11 galaxies in our sample also have detections in the $1 \mathrm{~mm}$ dust continuum. We can thus estimate the mass of the molecular gas independently of the $\mathrm{CO}$ data. The Rayleigh-Jeans part of the dust emission is only weakly dependent on the dust temperature, thus it can be used to trace the mass of dust. Using the dust-to-gas scaling (see, e.g., Sandstrom et al. 2013), it is possible to infer the gas mass via the dust mass.

Groves et al. (2015) compare CO-based gas masses with the monochromatic luminosity of the dust continuum in the Rayleigh-Jeans tail. Their analysis relies on a detailed study of 37 local spiral galaxies in the KINGFISH sample (Kennicutt et al. 2011). The galaxy luminosity in the Herschel/SPIRE $500 \mu \mathrm{m}$ band is found to scale almost linearly with the gas mass, yielding

$$
\frac{M_{\mathrm{gas}}}{10^{10} M_{\odot}}=28.5 \frac{\nu L_{\nu}(500 \mu \mathrm{m})}{10^{10} L_{\odot}} .
$$

We compute the rest-frame luminosity $\nu L_{\nu}(500 \mu \mathrm{m})$ from the observed $1 \mathrm{~mm}$ continuum of the galaxies in our sample. For the $k$-correction, we adopt a modified blackbody with $T_{\text {dust }}=25 \mathrm{~K}$ and $\beta=1.6$ (see, e.g., Beelen et al. 2006), shifted at the redshift of each source. Since the observing frequency $(242 \mathrm{GHz}$ ) falls close to the rest-frame $500 \mu \mathrm{m}$ (as most of our sources reside at $z \sim 1.2$ ), and we are sampling the RayleighJeans tail (which is almost insensitive to the dust temperature), the differences in the corrections due to the adopted templates are negligible for the purposes of this analysis. The adopted values for the $k$ correction, as well as the resulting gas masses, are listed in Table 4.

A similar approach was presented by Scoville et al. (2014, 2016). This calibration is tuned on a set of relatively massive $\left[(0.2-4) \times 10^{11} M_{\odot}\right]$ star-forming galaxies (30 local star-forming galaxies, 12 low-redshift ultraluminous IR galaxies (ULIRGs), and 30 SMGs at $z=1.4-3.0$ ), all having literature observations of the $\mathrm{CO}(1-0)$ transition. The tight relation observed between $\mathrm{CO}(1-0)$ luminosity and the restframe $850 \mu \mathrm{m}$ monochromatic luminosity (see Figure 1 in Scoville et al. 2016) suggests that a simple conversion factor can be used to derive gas masses from monochromatic dust continuum observations. By setting the dust temperature to $T_{\text {dust }}=25 \mathrm{~K}$ (following Scoville et al. 2014), we derive from Equation (12) in their paper $M_{\mathrm{ISM}}$ from our $1 \mathrm{~mm}$ flux densities as follows:

$$
\frac{M_{\mathrm{ISM}}}{10^{10} M_{\odot}}=\frac{1.20}{(1+z)^{4.8}} \frac{F_{\nu}}{\mathrm{mJy}}\left(\frac{\nu}{350 \mathrm{GHz}}\right)^{-3.8} \frac{\Gamma_{0}}{\Gamma_{\mathrm{RJ}}}\left(\frac{D_{\mathrm{L}}}{\mathrm{Gpc}}\right)^{2},
$$

where $F_{\nu}$ is the observed dust continuum flux density at the observing frequency $\nu(242 \mathrm{GHz}$ in our case $), D_{\mathrm{L}}$ is the luminosity distance, and $\Gamma_{\mathrm{RJ}}$ is a unitless correction factor that accounts for the deviation from the $\nu^{2}$ scaling of the RayleighJeans tail. In the reference sample of local galaxies, lowredshift ULIRGs, and high- $z$ SMGs that Scoville et al. (2014) used to calibrate Equation (3), $\Gamma_{\mathrm{RJ}}=\Gamma_{0}=0.71$. The resulting ISM masses are listed in Table 4.

Finally, we can infer an estimate of $M_{\text {gas }}$ from the estimate of the dust mass, $M_{\text {dust }}$, that we obtain via our MAGPHYS fit of the available SED, simply scaled by a fixed dust-to-gas mass ratio (DGR). Sandstrom et al. (2013) investigate the dust and gas content in a sample of local spiral galaxies and find $\mathrm{DGR} \approx 1 / 70$. Genzel et al. (2015) and Berta et al. (2016) perform a detailed analysis of both gas- and dust-mass estimates in galaxies at $0.9<z<3.2$ observed with Herschel, and find a lower value of DGR $\approx 1 / 100$, which is the value we adopt here. We stress that there is a factor $>2 \times$ scatter in the 


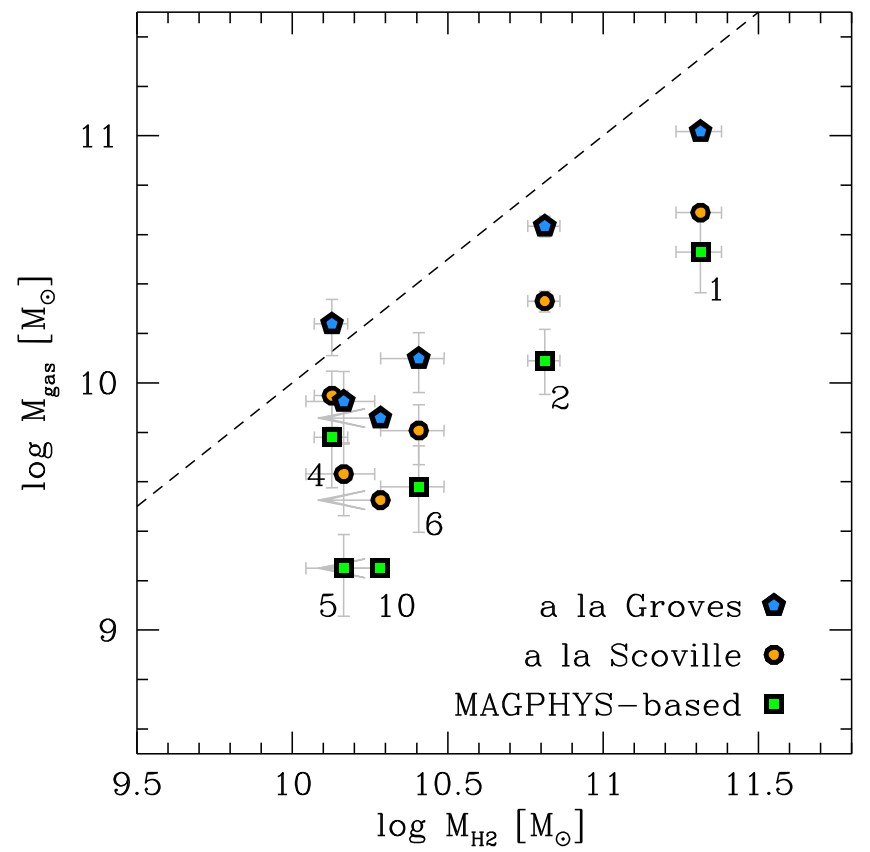

Figure 10. Comparison between the $\mathrm{H}_{2}$ masses that we derive from $\mathrm{CO}$ for the sources in our sample ( $x$-axis), and the gas masses inferred from the $1 \mathrm{~mm}$ continuum, following Equation (2) (Groves et al. 2015), Equation (3) (Scoville et al. 2014, 2016), and based on the MAGPHYS-based estimates of $M_{\text {dust }}$, assuming a dust-to-gas ratio of $1 / 100$ (Genzel et al. 2015) ( $y$-axis). The dashed line shows the one-to-one case. Only sources with a $1 \mathrm{~mm}$ continuum detection are shown. The dust-based estimates are correlated with each other as a result of the strong dependence on the $1 \mathrm{~mm}$ continuum emission. The various mass estimates are also correlated with the CO-based ones over 1.5 dex. There are systematic offsets among the various gas mass recipes, however, with dustbased masses that appear lower than those inferred from CO.

estimates of DGR owing to its dependence on $M_{*}$ and metallicity (Sandstrom et al. 2013; Berta et al. 2016). Following the fundamental metallicity relation in Mannucci et al. (2010), we estimate that galaxies in our sample typically have solar metallicities (the lowest metallicity estimates are for ID.9: $Z=0.6 \mathrm{Z}_{\odot}$; and ID.5: $Z=0.7 \mathrm{Z}_{\odot}$ ), therefore we do not foresee large intra-sample variations of DGR. For simplicity, in our analysis we thus assume a fixed DGR $=1 / 100$. While SED fits are available for all the galaxies in our sample, here we consider only those with a $1 \mathrm{~mm}$ detection, in order to best anchor the Rayleigh-Jeans tail of the dust emission. The resulting masses are listed in Table 4.

Figure 10 compares the gas estimates based on Equation (2), following Groves et al. (2015); those obtained via Equation (3), following Scoville et al. (2014); and the estimates based on dust from the MAGPHYS SED fits, with our CO-based estimate (assuming $\alpha_{\mathrm{CO}}=3.6 M_{\odot}\left[\mathrm{K} \mathrm{km} \mathrm{s}^{-1} \mathrm{pc}^{2}\right]^{-1}$ ). The dust-based gas estimates obtained with different approaches are strongly correlated with each other, as expected because they all scale (almost linearly) with $F_{\nu}(1 \mathrm{~mm})$. They also correlate well with the $\mathrm{CO}$-based $\mathrm{H}_{2}$ mass estimates over one and a half dex of dynamic range. However, systematic offsets are observed. The Groves et al. (2015) estimates are on average $1.5 \times$ lower than those based on CO. The estimates based on Scoville et al. (2016) are another $2 \times$ lower, and the masses based on MAGPHYS are a factor $1.7 \times$ lower than those obtained following Scoville et al. (2016).

What causes the discrepancies between these mass estimates? The $\mathrm{CO}$ masses might be overestimated because of our assumptions in terms of $\mathrm{CO}$ excitation and $\alpha_{\mathrm{CO}}$. A higher $\mathrm{CO}$ excitation would imply higher $r_{J 1}$, thus lower $\mathrm{CO}(1-0)$ luminosity (see Equation (1)). When we assume the M82 excitation template by Weiß et al. (2007) (see Figure 5), the inferred $M_{\mathrm{H} 2}$ masses would be $1.3 \times$ lower for ID.2-6, and $2.2 \times$ lower for ID.1 and ID.10. This would solve the discrepancy with respect to the estimates based on the Groves recipe, and it would mitigate, but not solve, the discrepancy with the other gas mass estimates. However, such a high $\mathrm{CO}$ excitation scenario is ruled out by our $1 \mathrm{~mm}$ line observations (see Figure 5). A lower value of $\alpha_{\mathrm{CO}}$ could also help. When we adopt the classical value for ULIRGs, $\alpha_{\mathrm{CO}}=$ $0.8 M_{\odot}\left(\mathrm{K} \mathrm{km} \mathrm{s}^{-1} \mathrm{pc}^{2}\right)^{-1}$ (Bolatto et al. 2013), the CO-based gas masses would be a factor 4.5 lower, thus in good agreement with those from the dust. Figure 6 shows that the majority of our sources lie along the relation of main-sequence galaxies/ local spiral galaxies in the $L_{\mathrm{IR}}-L_{\mathrm{CO}}^{\prime}$ plot. This is regardless of the choice of $\alpha_{\mathrm{CO}}$. Thorough studies of galaxies along this sequence support our choice for a higher value of $\alpha_{\mathrm{CO}}$ (e.g., Daddi et al. 2010a; Genzel et al. 2010, 2015; Sargent et al. 2014). Further support for our choice comes from the position of our sources along the "main sequence" of galaxies (Figure 1). Of the sources listed in Table 4 and appearing in Figure 10, only ID.5 could be considered a starburst in this respect. Adopting a lower $\alpha_{\mathrm{CO}}$ for only this source would lower its molecular gas mass by a factor $\sim 4.5$, thus bringing it close to the bulk of the "main-sequence" galaxies in terms of gas fraction (Figure 9), but pushing it away from the sequence in the star formation law plot (Figure 7). It would also reduce its depletion timescale (Figure 8) and bring the CO-based gas mass closer to the dust-based estimates (Figure 10). Similar considerations could also apply for ID.1, the CO-brightest galaxy in our sample. The compact morphology and the small separation from a companion galaxy, the rising $\mathrm{CO}$ emission at high $J$, the high values of $\Sigma_{\mathrm{SFR}}$ and $\Sigma_{\mathrm{H} 2}$, and the very high $M_{\mathrm{H} 2} / M_{*}$ all point toward a starburst scenario for this source; however, it is located along the main sequence of galaxies at $z \sim 2.5$ in Figure 1 , and the $L_{\mathrm{IR}}-L_{\mathrm{CO}(1-0)}^{\prime}$ plot (Figure 6) shows that this source is located along the sequence of local spirals and main-sequence galaxies (not along the sequence of starbursts), regardless of the choice of $\alpha_{\mathrm{CO}}$, even when we assume the extreme case of thermalized $\mathrm{CO}(3-2)$ emission in order to derive $L_{\mathrm{CO}(1-0)}^{\prime}$. Because of this, and because of the lack of any starburst signature (justifying a low $\left.\alpha_{\mathrm{CO}}\right)$ in all the other sources, the discrepancies between different gas mass estimates shown in Figure 10 cannot be mended solely by tuning our assumptions on the CO-based mass estimates.

The dust-based gas-mass estimates could also be affected by systematic uncertainties. The offset between the estimates based on Equations (2) and (3) suggests a systematic in the calibration of the two recipes. The luminosity range used in Groves et al. (2015) to derive Equation (2), e.g., does not cover the $>10^{10} L_{\odot}$ range, where our galaxies are found. Equation (3), based on Scoville et al. (2014), is pinned down to a longer wavelength than what is observed in ASPECS $(850 \mu \mathrm{m}$ in the rest-frame, instead of $\sim 500 \mu \mathrm{m})$, thus the $k$ correction is significant and dependent on the adopted dust template. In particular, Equation (3) explicitly assumes $\beta=1.8$, which might not be universally valid (see discussion in Paper II). Our dust SED is only poorly sampled. Most remarkably, the comparison between maps of the $\mathrm{CO}$ and dust emission in ID.2 

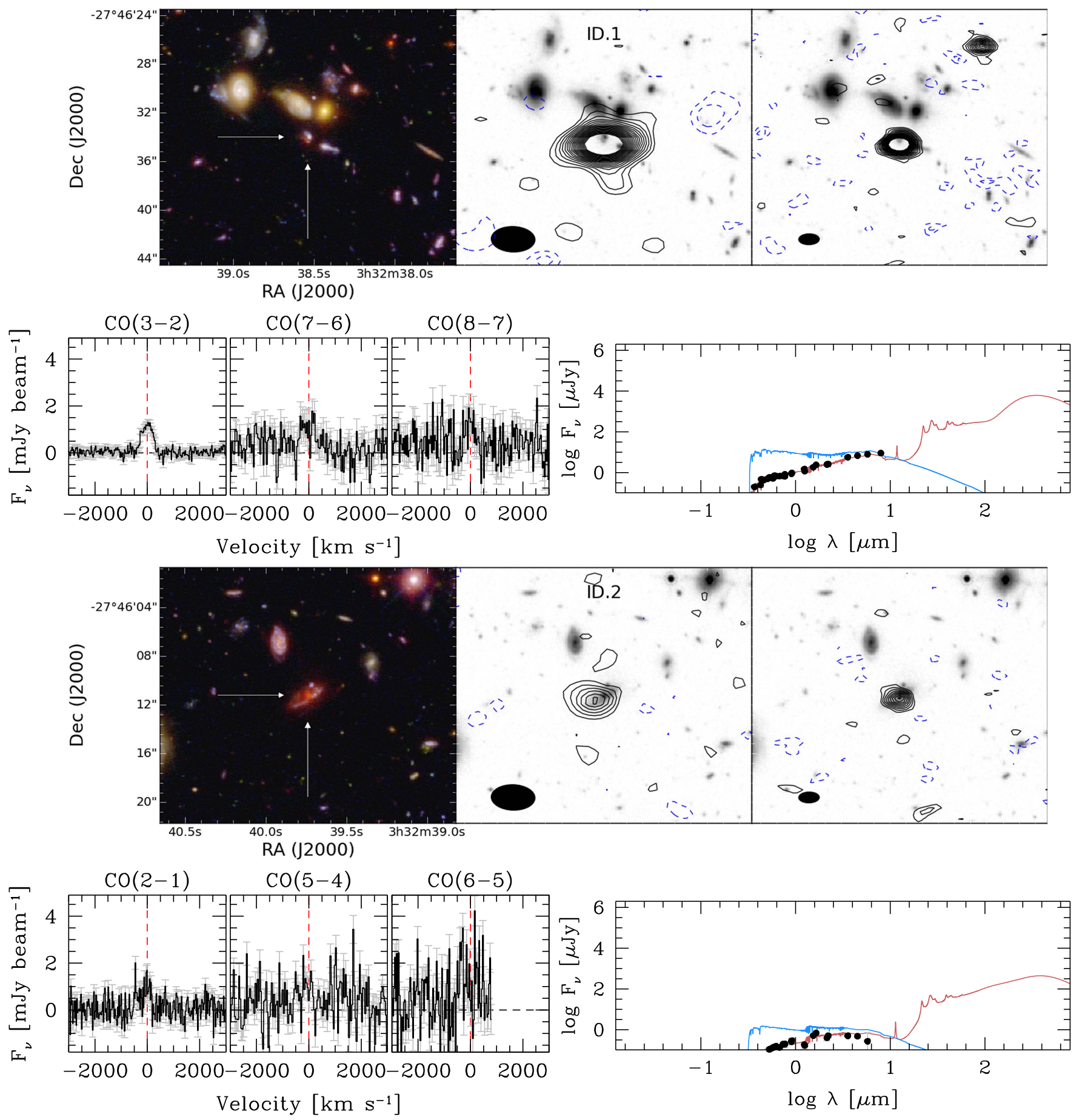

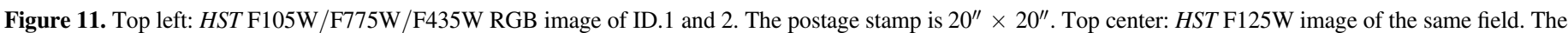

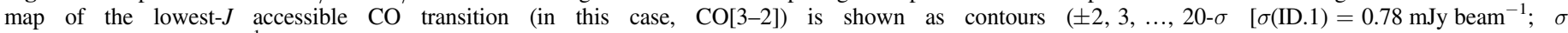

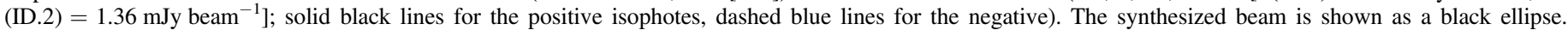

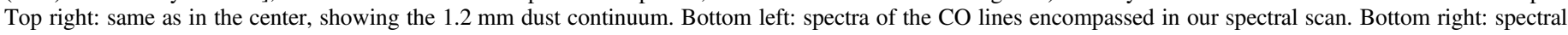

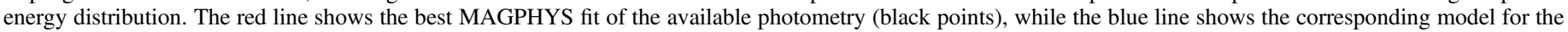
unobscured stellar component. The main output parameters are quoted.

suggests that the gas is optically thick over a large area, while the dust is not. We might be missing part of the dust emission as a result of the surface brightness limits, thus affecting our estimates of the total ISM mass. In ID.3, the dust continuum emission is not detected at all, despite the bright $\mathrm{CO}$ emission. Since we do not detect any significant $1 \mathrm{~mm}$ continuum associated with the extended disk of ID.2, and no dust emission at all in ID.3, it is hard to assess how large a correction we should consider. It is possible that a similar issue is present in other sources, in particular in galaxies that we see as CO emitters, but for which we do not recover any $1 \mathrm{~mm}$ continuum emission (see, e.g., Figure 6). Finally, the underlying assumption in the dust-based gas estimates is the dust-to-gas ratio. This can change significantly as a function of metallicity and of 

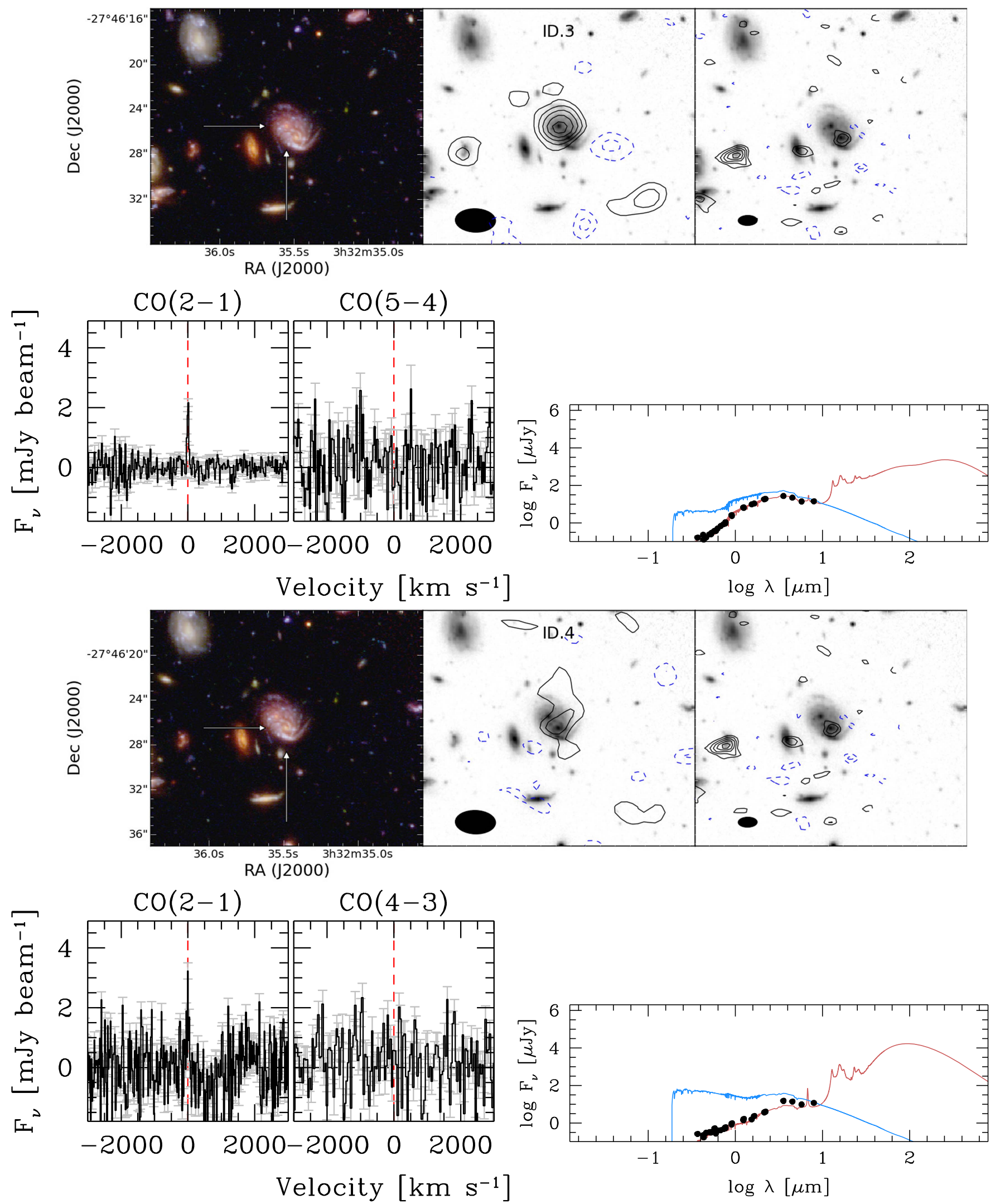

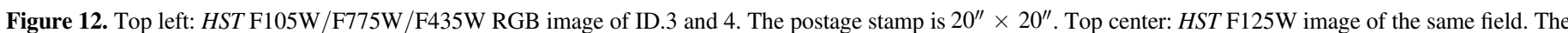

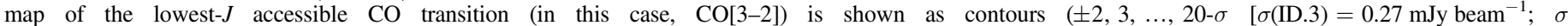

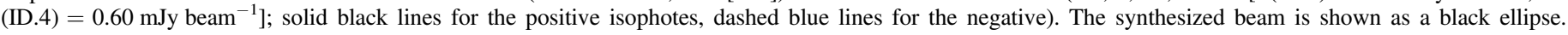

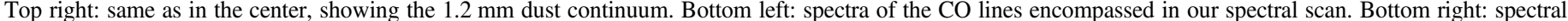

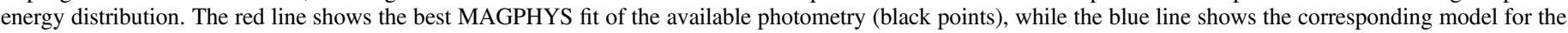
unobscured stellar component. The main output parameters are quoted. 

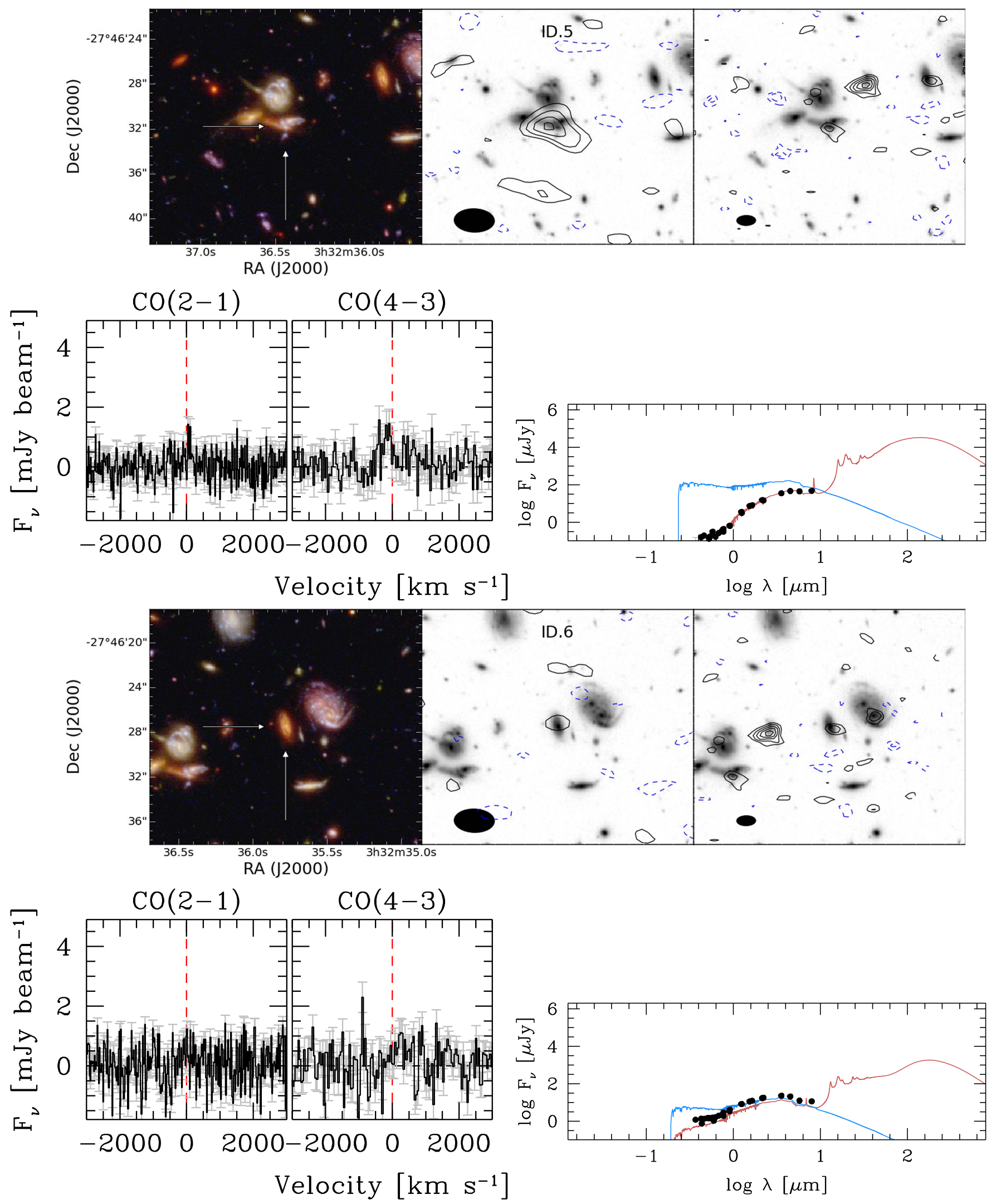

Figure 13. Top left: HST F105W/F775W/F435W RGB image of ID.5 and 6. The postage stamp is $20^{\prime \prime} \times 20^{\prime \prime}$. Top center: HST F125W image of the same field. The map of the lowest- $J$ accessible $\mathrm{CO}$ transition (in this case, $\mathrm{CO}[3-2])$ is shown as contours $\left( \pm 2,3, \ldots, 20-\sigma\left[\sigma(\mathrm{ID} .5)=1.13 \mathrm{mJy}\right.\right.$ beam ${ }^{-1}$; $\sigma$ (ID.6) $=0.79 \mathrm{mJy} \mathrm{beam}^{-1}$ ]; solid black lines for the positive isophotes, dashed blue lines for the negative). The synthesized beam is shown as a black ellipse. Top right: same as in the center, showing the $1.2 \mathrm{~mm}$ dust continuum. Bottom left: spectra of the CO lines encompassed in our spectral scan. Bottom right: spectral energy distribution. The red line shows the best MAGPHYS fit of the available photometry (black points), while the blue line shows the corresponding model for the unobscured stellar component. The main output parameters are quoted. 



\section{Velocity $\left[\mathrm{km} \mathrm{s}^{-1}\right]$}

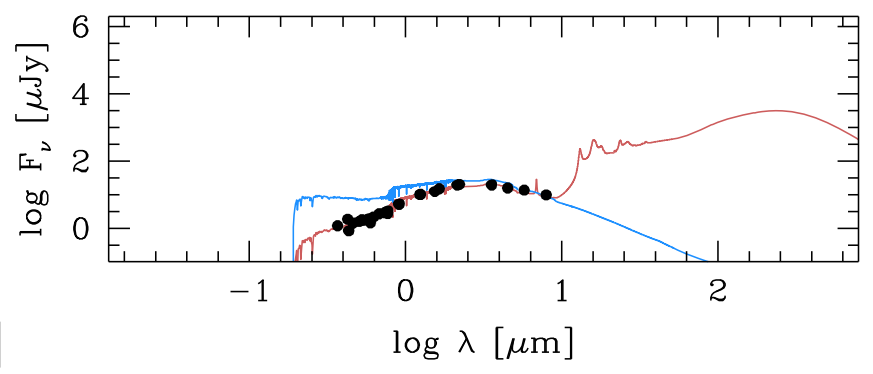

Figure 14. Top left: HST F105W/F775W/F435W RGB image of ID.7 and 8 . The postage stamp is $20^{\prime \prime} \times 20^{\prime \prime}$. Top center: HST F125W image of the same field. The map of the lowest $J$ accessible $\mathrm{CO}$ transition (in this case, $\mathrm{CO}[3-2])$ is shown as contours $\left( \pm 2,3, \ldots, 20-\sigma \quad\left[\sigma(\mathrm{ID} .7)=0.67 \mathrm{mJy}^{2}\right.\right.$ beam ${ }^{-1}$; $\sigma$ (ID.8) $=1.18 \mathrm{mJy} \mathrm{beam}^{-1}$ ]; solid black lines for the positive isophotes, dashed blue lines for the negative). The synthesized beam is shown as a black ellipse. Top right: same as in the center, showing the $1.2 \mathrm{~mm}$ dust continuum. Bottom left: spectra of the CO lines encompassed in our spectral scan. Bottom right: spectral energy distribution. The red line shows the best MAGPHYS fit of the available photometry (black points), while the blue line shows the corresponding model for the unobscured stellar component. The main output parameters are quoted. 

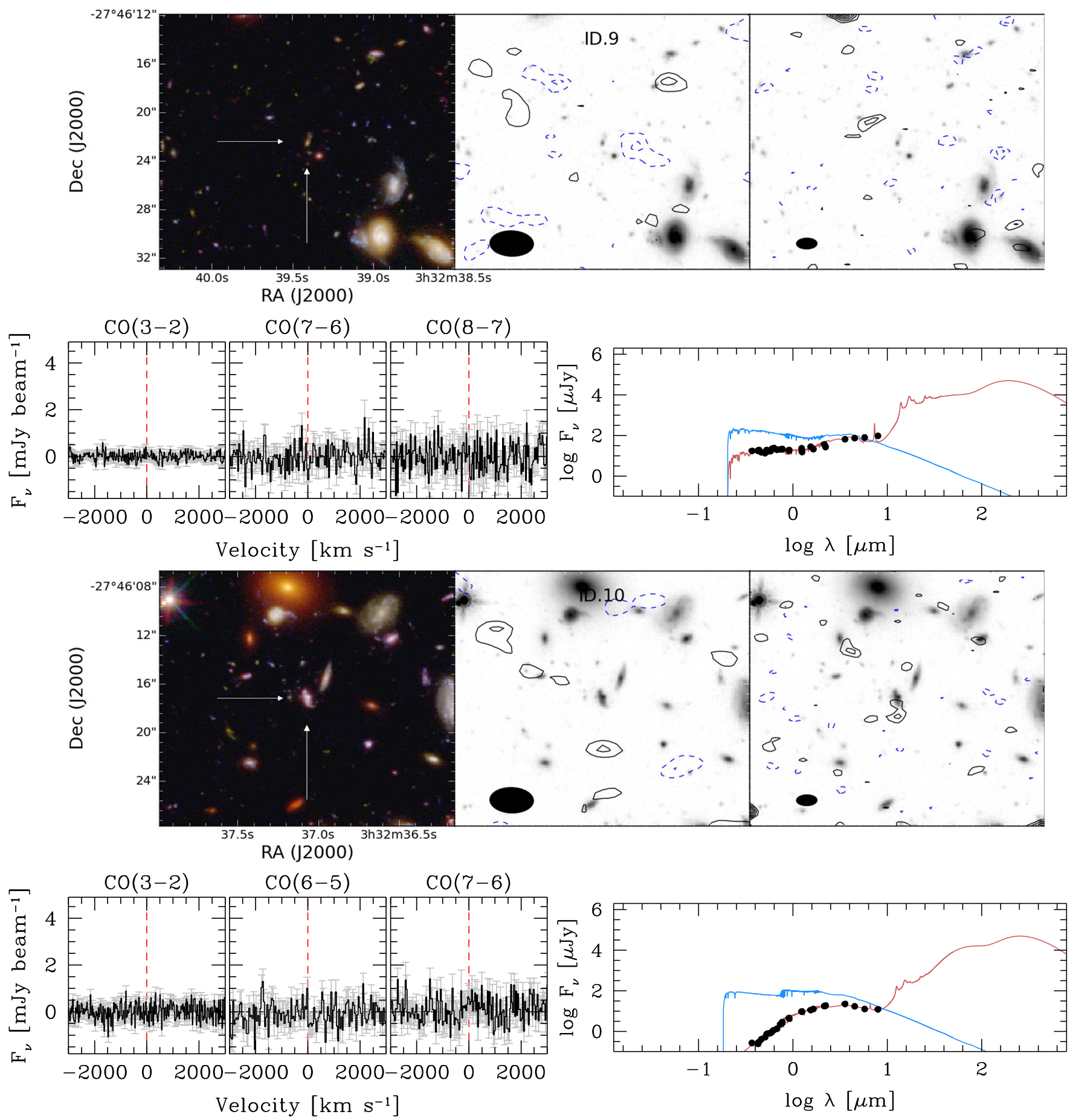

Figure 15. Top left: HST F105W/F775W/F435W RGB image of ID.9 and 10. The postage stamp is $20^{\prime \prime} \times 20^{\prime \prime}$. Top center: HST F125W image of the same field. The map of the lowest $J$ accessible $\mathrm{CO}$ transition (in this case, $\mathrm{CO}[3-2])$ is shown as contours $\left( \pm 2,3, \ldots, 20-\sigma \quad\left[\sigma(\mathrm{ID} .9)=0.44 \mathrm{mJy}^{2}\right.\right.$ beam ${ }^{-1}$; $\sigma$ (ID.10) $=0.64 \mathrm{mJy} \mathrm{beam}^{-1}$ ]; solid black lines for the positive isophotes, dashed blue lines for the negative). The synthesized beam is shown as a black ellipse. Top right: same as in the center, showing the $1.2 \mathrm{~mm}$ dust continuum. Bottom left: spectra of the CO lines encompassed in our spectral scan. Bottom right: spectral energy distribution. The red line shows the best MAGPHYS fit of the available photometry (black points), while the blue line shows the corresponding model for the unobscured stellar component. The main output parameters are quoted.

other parameters in the galaxy (see Sandstrom et al. 2013, for a detailed discussion). A lower value of DGR (e.g., DGR $1 / 200$ ) would halve the discrepancy between the MAGPHYSbased estimates and the CO-based ones. While this is a possibility at the low-mass end of Figure 10, we point out that the relatively high stellar mass of the galaxies at the bright end supports metallicity values close to solar, thus disfavoring the high DGR values needed to reconcile the two gas-mass estimates.

\section{SUMMARY}

We present a study of the molecular gas properties as derived from $\mathrm{CO}$ observations of high-redshift galaxies in ASPECS, 

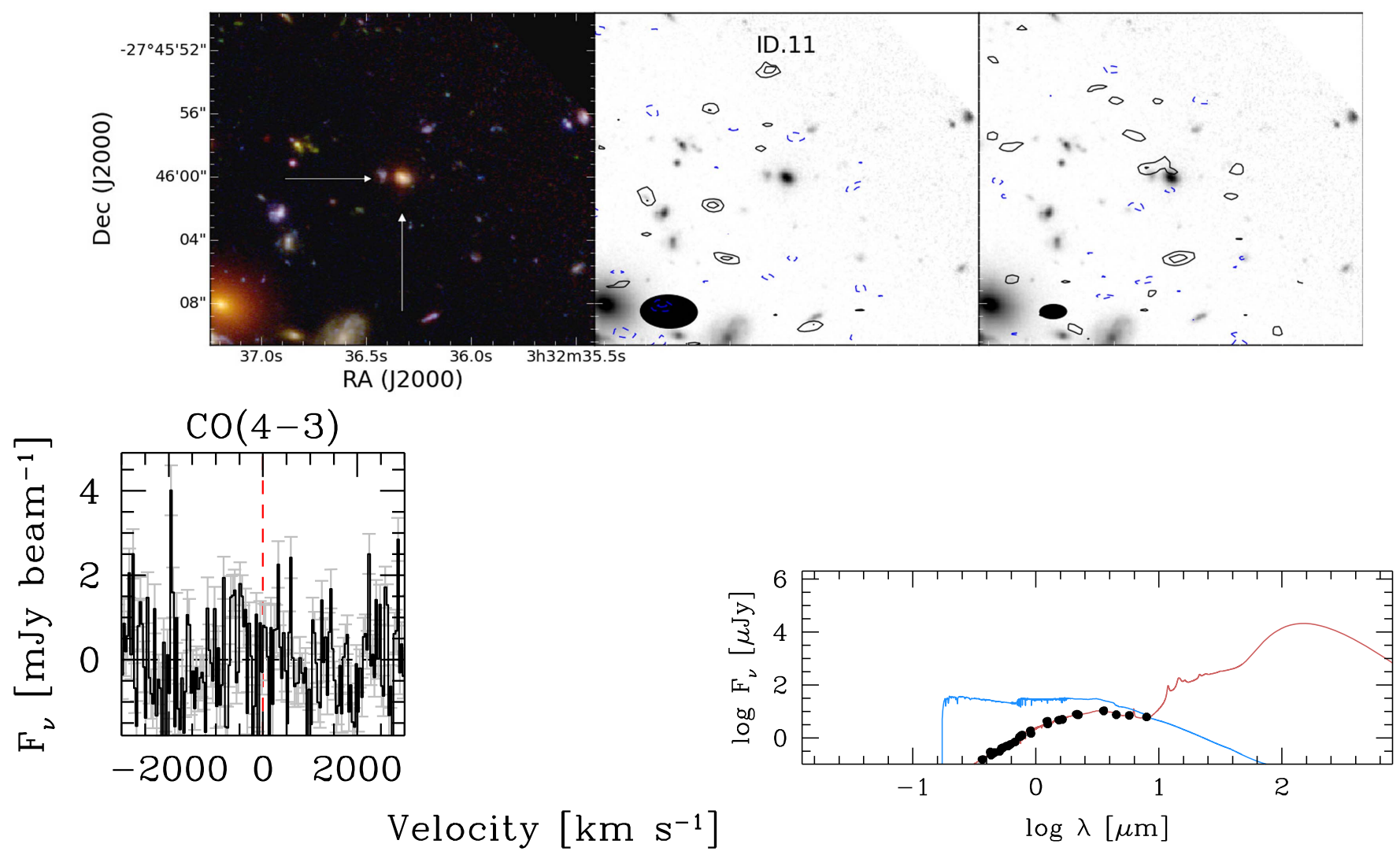

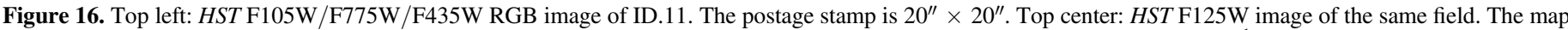

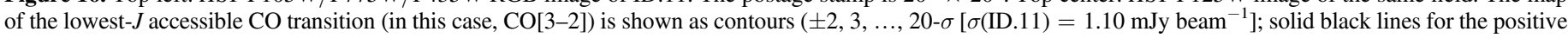

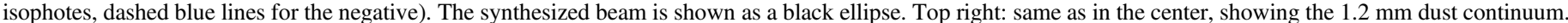

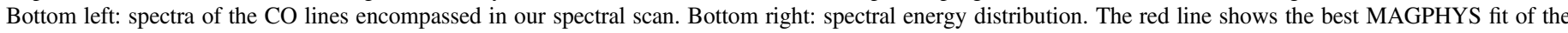

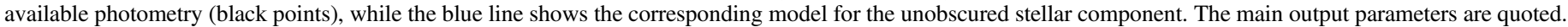

the ALMA Spectroscopic Survey in the Hubble UDF. This data set consists of a blind survey of molecular gas in the ALMA 3 and $1 \mathrm{~mm}$ bands targeting a region with the deepest HST imaging available, the so-called XDF. Our observations cover hundreds of high-redshift galaxies with well-characterized SEDs, i.e., we can test our expectations in terms of molecular gas content in galaxies without any prior selection through their optical or near-IR properties. This allows us to analyze our CO measurements and limits in the context of the global properties of the associated galaxies, thanks to an exquisite wealth of ancillary multi-wavelength information.

We focus on the galaxies for which a secure redshift is available, either via our ASPECS CO observations or from optical/near-IR spectroscopy reported in the literature. In particular, we consider those sources for which our sophisticated fit of the optical-to-mid-IR SED implies high-IR luminosity $\left(L_{\mathrm{IR}}>10^{11} L_{\odot}\right)$. These galaxies are expected to be detected in $\mathrm{CO}$ based on the empirical relation between $\mathrm{CO}$ and dust luminosity. We also restrict our analysis to those galaxies with a redshift such that a CO transition with $J_{\text {up }}<5$ is covered in our ASPECS observations.

Success of $\mathrm{CO}$ detection. Out of 11 sources selected in this way, 4 are also identified in our CO blind search (see Paper I of this series). Three additional galaxies are detected in $\mathrm{CO}$, although with lower significance. The faintest galaxy detected in $\mathrm{CO}$ (at $\sim 3 \sigma$ level) harbors an AGN. This most likely leads to an overestimate of the IR luminosity in our analysis (if the AGN component contributes significantly to the rest-frame
optical/UV emission). Finally, four sources remain undetected in CO. In two of them, the lack of $\mathrm{CO}$ detection might be attributed to $\mathrm{CO}$ excitation, as the lowest $J$ transition that we targeted in these sources is the $\mathrm{CO}(4-3)$ line. This would point toward a very low-excitation scenario for these two sources, however. The other two undetected galaxies are just above our IR luminosity cut. They reside at relatively high redshift $(z=2.0-2.5)$. In these cases, the lack of a detection might be attributed to the insufficient depth of our ASPECS observations, and/or to modest $\mathrm{CO}$ excitation.

$\mathrm{CO}$ excitation. As we cover $\mathrm{CO}$ emission in two separate ALMA bands, we constrain the $\mathrm{CO}$ excitation in all of our COdetected galaxies. In no case do we find evidence of high excitation, as observed in the center of M82 or in IR-luminous SMGs or QSOs at high redshift. The galaxy that has the highest excitation is a bright compact galaxy, showing high SFR surface density (ID.1). We attribute the high $\mathrm{CO}$ excitation to either the increased radiation density (due to the locally intense star formation) or to the high density of the gas. A second source (ID.5) shows CO excitation slightly higher than the average "main sequence" galaxy; this is consistent with this galaxy being a starburst, as suggested by other indicators (sSFR with respect to the "main sequence" at that redshift; IR-to-CO luminosity ratio; depletion time; etc.). On the other hand, $\mathrm{CO}$ excitation is typically very low, often consistent with or even lower than Milky Way excitation at least up to $J=5$. In one case, an $r_{52}$ value as low as $<0.025$ was measured (for comparison, in the Milky Way we have $r_{52}=0.16$ ). An X-ray 
bright AGN with an extended gas reservoir (ID.2) also shows modest $\mathrm{CO}$ excitation; in this case, any effect that the AGN may have in the center is diminished by the extended molecular gas emission in the disk. Interestingly, the CO-brightest galaxy in our sample, ID.1, is also detected in the X-rays. Its X-ray luminosity is modest, however, and roughly consistent with the extrapolation of the SFR $-L_{\mathrm{X}}$ relation observed in local starbursts (Ranalli et al. 2003).

Location with respect to the "main sequence". We discuss our findings in the context of previous molecular gas observations at high redshift (star formation law, gas depletion times, gas-mass fractions), based on sophisticated SED modeling of their multi-wavelength properties using the highredshift extension of the MAGPHYS code. Half of the galaxies in our sample reside on the "main sequence" of star-forming galaxies at their redshift. Three galaxies are found in the starburst region (although in one of them the SFR might be overestimated due to the contamination from an optically bright AGN). Finally, two sources are found below the main sequence, suggesting that they are more quiescent systems.

The "star formation law". To first order, the CO-detected galaxies in the UDF cover the same parameter space as previous galaxy samples in the $L_{\mathrm{IR}}-L_{\mathrm{CO}}^{\prime}$ diagram, although they preferentially reside toward the low-IR luminosity envelope of the relation, along the same sequence of local spiral galaxies and close to color-selected galaxies at $z>1$. Only two CO-detected sources lie on the opposite side, closer to the locus of high- $z$ starbursts. Two of the CO non-detections are found to be inconsistent with the $L_{\mathrm{IR}}-L_{\mathrm{CO}}^{\prime}$ relation, suggesting that $\mathrm{CO}$ excitation in these sources must be low. Using HST imaging to derive the scale radii of the galaxies in our sample, we discuss their location in the "star formation law" diagram: on average, the sources agree with a depletion time of $\sim 1$ Gyr, as found in previous studies, but outliers (up to 1 dex) exist.

Gas fractions. With only two remarkable exceptions, the gas fractions observed in our study are slightly lower than what is found in targeted observations of main-sequence galaxies at similar redshift, but still significantly higher than what is typically observed in the local universe.

$C O$-versus dust-based estimates of gas mass. In a few cases, we have gas mass estimates derived from $\mathrm{CO}$ as well as the dust continuum, via different recipes involving the dust-to-gas ratio. The dust-based estimates are a factor of $\sim 2-5$ smaller than those based on $\mathrm{CO}$. This is consistent with recent reports in the literature that dust-based estimates of ISM masses of main-sequence galaxies give significantly lower values than using the $\mathrm{CO}$ emission. All these methods use a number of assumptions: $\mathrm{CO}$ : extrapolation to a $\mathrm{CO}(1-0)$ flux from a higher- $J$ transition + choice of $\mathrm{CO}-$ to- $\mathrm{H}_{2}$ conversion factor, dust: assumption of temperature, dust SED template, optical depth, and dust-to-gas ratio. A larger sample of galaxies with well-defined dust SEDs is required to ultimately decide which gas-mass estimator is preferred.

In summary, accounting for detections as well as nondetections, we find large variations in the molecular gas properties of high-redshift galaxies. This might reflect the large variations in gas content of disk galaxies seen in semianalytical models (see, e.g., Lagos et al. 2011 and Figure 9 in Popping et al. 2014). Perhaps not unexpectedly, global scaling relations cannot account for the large variations in gas content in individual high-redshift galaxies. Our approach through blind frequency scans of well-characterized cosmological deep fields adds additional constraints to the studies of the molecular gas content in distant galaxies, and the scans are thus complementary to dedicated studies of single galaxies that are pre-selected by their optical properties (e.g., SFR and stellar mass). Our study demonstrates that such studies are now feasible, even with early-cycle ALMA observations. Now that ALMA has reached completion, similar studies of larger fields will result in large statistical samples, which are required to fully understand and beat down systematics and small number statistics. This will provide us with an entirely new approach for characterizing the molecular gas content in distant galaxies.

We thank the anonymous referee for the positive feedback and useful comments. R.D. thanks Laura Zschaechner for insightful discussions. F.W., I.R.S., and R.J.I. acknowledge support through ERC grants COSMIC-DAWN, DUSTYGAL, and COSMICISM, respectively. M.A. acknowledges partial support from FONDECYT through grant 1140099. D.R. acknowledges support from the National Science Foundation under grant number AST-1614213 to Cornell University. F.E.B. and L.I. acknowledge Conicyt grants Basal-CATA PFB-06/2007 and Anilo ACT1417. F.E.B. also acknowledge support from FONDECYT Regular 1141218 (FEB), and the Ministry of Economy, Development, and Tourism's Millennium Science Initiative through grant IC120009, awarded to The Millennium Institute of Astrophysics, MAS. I.R.S. also acknowledges support from STFC (ST/L00075X/1) and a Royal Society/Wolfson Merit award. Support for R.D. and B.M. was provided by the DFG priority program 1573 "The physics of the interstellar medium." A.K. and F.B. acknowledge support by the Collaborative Research Council 956, subproject A1, funded by the Deutsche Forschungsgemeinschaft (DFG). PI acknowledges Conict grants Basal-CATA PFB-06/ 2007 and Anilo ACT1417. R.J.A. was supported by FONDECYT grant number 1151408. This paper makes use of the following ALMA data: [ADS/JAO.ALMA\#2013.1.00146.S and 2013.1.00718.S.]. ALMA is a partnership of ESO (representing its member states), NSF (USA) and NINS (Japan), together with NRC (Canada), NSC and ASIAA (Taiwan), and KASI (Republic of Korea), in cooperation with the Republic of Chile. The Joint ALMA Observatory is operated by ESO, AUI/NRAO and NAOJ. The $3 \mathrm{~mm}$ part of ALMA project has been supported by the German ARC.

\section{REFERENCES}

Aravena, M., Carilli, C. L., Salvato, M., et al. 2012, MNRAS, 426, 258 Aravena, M., Decarli, R., Walter, F., et al. 2016a, ApJ, 833, 68 (Paper II) Aravena, M., Decarli, R., Walter, F., et al. 2016b, ApJ, 833, 71 (Paper V) Aravena, M., Spilker, J. S., Bethermin, M., et al. 2016, MNRAS, 457, 4406 Beckwith, S. V., Stiavelli, M., Koekemoer, A. M., et al. 2006, AJ, 132, 1729 Beelen, A., Cox, P., Benford, D. J., et al. 2006, ApJ, 642, 694

Berta, S., Lutz, D., Genzel, R., Förster-Schreiber, N. M., \& Tacconi, L. J. 2016, A\&A, 587A, 73

Bertoldi, F., Cox, P., Neri, R., et al. 2003, A\&A, 409, L47

Béthermin, M., Daddi, E., Magdis, G., et al. 2015, A\&A, 573, 113 Bolatto, A. D., Wolfire, M., \& Leroy, A. K. 2013, ARA\&A, 51, 207

Bothwell, M. S., Smail, I., Chapman, S. C., et al. 2013, MNRAS, 429, 3047 Bouwens, R., Aravena, M., Decarli, R., et al. 2016, ApJ, 833, 72 (Paper VI) Carilli, C., Chluba, J., Decarli, R., et al. 2016, ApJ, 833, 73 (Paper VII) Carilli, C. L., \& Walter, F. 2013, ARA\&A, 51, 105

Casey, C. M., Chapman, S. C., Neri, R., et al. 2011, MNRAS, 415, 2723

Casey, C. M., Narayanan, D., \& Cooray, A. 2014, PhRv, 541, 45

Chabrier, G. 2003, PASP, 115, 763

Chapman, S. C., Bertoldi, F., Smail, I., et al. 2015a, MNRAS, 449, L68 
Chapman, S. C., Bertoldi, F., Smail, I., et al. 2015b, MNRAS, 453, 951

Chapman, S. C., Neri, R., Bertoldi, F., et al. 2008, ApJ, 689, 889

Coe, D., Benítez, N., Sánchez, S. F., et al. 2006, AJ, 132, 926

da Cunha, E., Charlot, S., \& Elbaz, D. 2008, MNRAS, 388, 1595

da Cunha, E., Walter, F., Smail, I. R., et al. 2015, ApJ, 806, 110

Daddi, E., Bournaud, F., Walter, F., et al. 2010a, ApJ, 713, 686

Daddi, E., Dannerbauer, H., Liu, D., et al. 2015, A\&A, 577, 46

Daddi, E., Elbaz, D., Walter, F., et al. 2010b, ApJL, 714, L118

Decarli, R., Walter, F., Aravena, M., et al. 2016a, ApJ, 833, 69 (Paper III)

Decarli, R., Walter, F., Carilli, C., et al. 2014, ApJ, 782, 78

Draine, B. T., \& Lee, H. M. 1984, ApJ, 285, 89

Elbaz, D., Daddi, E., Le Borgne, D., et al. 2007, A\&A, 468, 33

Elbaz, D., Dickinson, M., Hwang, H. S., et al. 2011, A\&A, 533, 119

Evans, I. N., Primini, F. A., Glotfelty, K. J., et al. 2010, ApJS, 189, 37

Förster Schreiber, N. M., Genzel, R., Bouché, N., et al. 2009, ApJ, 706, 1364

Frayer, D. T., Ivison, R. J., Scoville, N. Z., et al. 1998, ApJL, 506, L7

Geach, J. E., Smail, I., Moran, S. M., et al. 2011, ApJL, 730, L19

Genzel, R., Tacconi, L. J., Gracia-Carpio, J., et al. 2010, MNRAS, 407, 2091

Genzel, R., Tacconi, L. J., Lutz, D., et al. 2015, ApJ, 800, 20

Greve, T. R., Bertoldi, F., Smail, I., et al. 2005, MNRAS, 359, 1165

Groves, B. A., Schinnerer, E., Leroy, A., et al. 2015, ApJ, 799, 96

Hodge, J. A., Riechers, D., Decarli, R., et al. 2015, ApJL, 798, L18

Illingworth, G. D., Magee, D., Oesch, P. A., et al. 2013, ApJS, 209, 6

Kennicutt, R. C. 1998, ApJ, 498, 541

Kennicutt, R. C., Calzetti, D., Aniano, G., et al. 2011, PASP, 123, 1347

Kennicutt, R. C., \& Evans, N. J. 2012, ARA\&A, 50, 531

Kurk, J., Cimatti, A., Daddi, E., et al. 2013, A\&A, 549, 63

Lagos, C. d. P., Bayet, E., Baugh, C. M., et al. 2011, MNRAS, 418, 1649

Le Fèvre, O., Vettolani, G., Garilli, B., et al. 2005, A\&A, 439, 845

Lehmer, B. D., Brandt, W. N., Alexander, D. M., et al. 2005, ApJS, 161, 21

Leroy, A. K., Walter, F., Brinks, E., et al. 2008, AJ, 136, 2782

Magdis, G. E., Daddi, E., Elbaz, D., et al. 2011, ApJL, 740, L15

Magdis, G. E., Daddi, E., Sargent, M., et al. 2012, ApJL, 758, L9

Magnelli, B., Saintonge, A., Lutz, D., et al. 2012, A\&A, 548, 22

Mannucci, F., Cresci, G., Maiolino, R., Marconi, A., \& Gnerucci, A. 2010, MNRAS, 408, 2115

Meijerink, R., Spaans, M., \& Israel, F. P. 2007, A\&A, 461, 793

Momcheva, I. G., Brammer, G. B., van Dokkum, P. G., et al. 2016, ApJS, 225,27
Morris, A. M., Kocevski, D. D., Trump, J. R., et al. 2015, AJ, 149, 178

Narayanan, D., \& Krumholz, M. R. 2014, MNRAS, 442, 1411

Neri, R., Genzel, R., Ivison, R. J., et al. 2003, ApJL, 597, L113

Noeske, K. G., Weiner, B. J., Faber, S. M., et al. 2007, ApJL, 660, L43

Oke, J. B., \& Gunn, J. E. 1983, ApJ, 266, 713

Planck Collaboration XIII 2016, A\&A, 594A, 13

Popping, G., Somerville, R. S., \& Trager, S. C. 2014, MNRAS, 442, 2398

Ranalli, P., Comastri, A., \& Setti, G. 2003, A\&A, 399, 39

Riechers, D. A., Bradford, C. M., Clements, D. L., et al. 2013, Natur, 496, 329

Riechers, D. A., Carilli, C. L., Walter, F., \& Momjian, E. 2010, ApJL, 724, L153

Riechers, D. A., Hodge, J., Walter, F., Carilli, C. L., \& Bertoldi, F. 2011, ApJL, 739, L31

Saintonge, A., Lutz, D., Genzel, R., et al. 2013, ApJ, 778, 2

Sandstrom, K. M., Leroy, A. K., Walter, F., et al. 2013, ApJ, 777, 5

Santini, P., Maiolino, R., Magnelli, B., et al. 2014, A\&A, 562, 30

Sargent, M. T., Daddi, E., Béthermin, M., et al. 2014, ApJ, 793, 19

Schmidt, M. 1959, ApJ, 129, 243

Schreiber, C., Pannella, M., Elbaz, D., et al. 2015, A\&A, 575, 74

Schruba, A., Leroy, A. K., Walter, F., et al. 2011, AJ, 142, 37

Scoville, N., Aussel, H., Sheth, K., et al. 2014, ApJ, 783, 84

Scoville, N., Sheth, K., Aussel, H., et al. 2016, ApJ, 820, 83

Silverman, J. D., Daddi, E., Rodighiero, G., et al. 2015, ApJL, 812, L23

Skelton, R. E., Whitaker, K. E., Momcheva, I. G., et al. 2014, ApJS, 214, 24

Spilker, J. S., Aravena, M., Marrone, D. P., et al. 2015, ApJ, 811, 124

Spilker, J. S., Marrone, D. P., Aguirre, J. E., et al. 2014, ApJ, 785, 149

Tacconi, L. J., Genzel, R., Neri, R., et al. 2010, Natur, 463, 781

Tacconi, L. J., Neri, R., Genzel, R., et al. 2013, ApJ, 768, 74

van der Wel, A., Bell, E. F., Häussler, B., et al. 2012, ApJS, 203, 24

Walter, F., Bertoldi, F., Carilli, C., et al. 2003, Natur, 424, 406

Walter, F., Decarli, R., Aravena, M., et al. 2016, ApJ, 833, 67 (Paper I)

Wang, R., Wagg, J., Carilli, C. L., et al. 2013, ApJ, 773, 44

Weiß, A., Downes, D., Neri, R., et al. 2007, A\&A, 467, 955

Whitaker, K. E., van Dokkum, P. G., Brammer, G., \& Franx, M. 2012, ApJL, 754, L29

Wuyts, S., Förster Schreiber, N. M., Lutz, D., et al. 2011, ApJ, 738, 106

Xue, Y. Q., Luo, B., Brandt, W. N., et al. 2011, ApJS, 195, 10 